\title{
Asymptotic Solution for the Current Profile of Passive Bare Electrodynamic Tethers
}

\author{
Claudio Bombardelli, ${ }^{*}$ Jesus Peláez, ${ }^{\dagger}$ and Manuel Sanjurjo ${ }^{\ddagger}$ \\ Technical University of Madrid, 28040 Madrid, Spain
}

\begin{abstract}
A relatively high-accuracy analytical solution for the current and potential profile along a passive bare electrodynamic tether is provided using perturbation theory. An ad hoc nondimensional formulation of the governing local bias and orbital motion limited current collection equations allows one to approach the problem with a perturbation technique in which a parameter, epsilon, quantifies the influence of ohmic effects on the final solution. For the case of small ohmic effects an approximate solution is obtained with a third-order expansion. Conversely, the case of dominant ohmic effects is treated based on an extension of the exact analytical solution available for the particular case of zero load and negligible potential drop at the cathodic end of the tether. After computing the analytical current and potential profile the maximum and average current, the Lorentz force and torque, as well as the optimum load impedance for maximum power generation are obtained. When compared with the exact, numerically-computed solution an accuracy of better than $5 \%$ is achieved for the computation of the average current across the full parameter space. The error with respect to the generated power becomes negligible when the load impedance is set to the optimum value, while it can grow to a maximum of about $30 \%$ for the less relevant case in which the load impedance of the power generation system is badly mismatched. The results, which are valid for a general rectilinear passive electrodynamic tether with constant cross section satisfying orbital motion limited theory and irrespective of the particular orbit configuration, will be of aid in the design and analysis of space missions involving bare electrodynamic tethers.
\end{abstract}

\section{Nomenclature}

$=$

$=$

$=$

$=$

$=$

line

Lorentz force

$=$ maximum tether current current

$=$ nondimensional current

$=$ tether length

$=$ ion mass

$=$ electron plasma density

$=$ tether cross-section perimeter section

$=$ orbit radius

$=$ planet radius
$=$ anodic and cathodic segment current, respectively

$=$ maximum zero-ohmic-effect current

$=$ modified beyond orbital motion limited characteristic

$=$ nondimensional current (traditional formulation)

$=$ nondimensional average current

$=$ nondimensional anodic and cathodic current

$=$ nondimensional short-circuit current

$=$ tether characteristic length (traditional formulation)

$=$ electron mass, $9.10938188 \times 10^{-31} \mathrm{~kg}$

$=$ electron charge, $1.60217646 \times 10^{-19} \mathrm{C}$

$=$ equivalent radius of electrodynamic tether cross

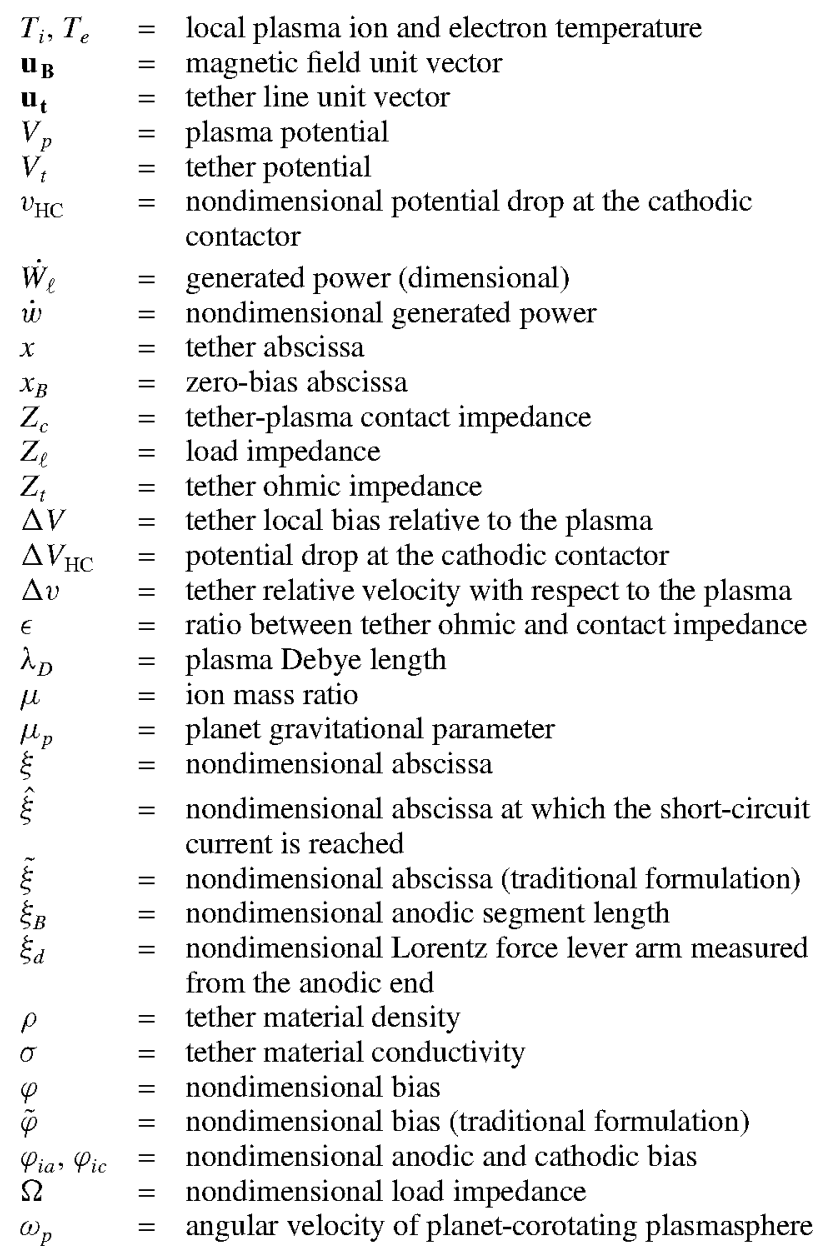

\section{Introduction}

$\mathbf{T}$ HE bare electrodynamic tether (EDT) concept was first introduced in 1991 by Sanmartin et al. [1] to tackle the problem 
of limited electron collection in high-power EDTs. In essence, having the uninsulated tether working as electron-collecting anode allowed to get rid of both space charge limits and magnetic guiding effects affecting big conductive spheres previously used as anodic contactors. The improvement in performance with respect to previous design solutions raised the interest of the space community across a broad spectrum of applications. An exhaustive description of the concept with its main applications can be found in [2] together with a large selection of references.

When working in passive mode a bare EDT system requires not more than an uninsulated conductive wire kept straight mainly by gravity gradient (hanging tether) or by centrifugal forces (spinning tethers) and collecting electrons from the surrounding plasmasphere, as well as one (or more) cathodic plasma contactor conveniently placed at the tether end(s) to expel electrons back to the plasma. For sufficiently long tethers a relatively high current can be obtained with no need for active onboard power supplies. Power can instead be generated on board by having a useful load (a battery, a measurement instrument, a transmitter, etc.) interposed between the tether and the cathodic plasma contactor. Proper matching of the load impedance with the EDT current and voltage characteristics allows to maximize such power.

As it emerges from the literature, the main aspects of bare electrodynamic tether design and optimization both in the generator and thruster mode were already addressed and solved since the publication of the first two articles on the subject $[1,3]$. Among the important conclusions of this early work emerges that the best use of the tether mass for satellite deorbiting and power generation is achieved by increasing tether length while decreasing cross section. For deorbiting applications in Earth orbit this corresponds to EDTs in which the potential drop due to ohmic losses along the tether has a dominant role (dominant ohmic effects regime). Conversely the generation of power at the expense of orbital energy is maximum in the small-ohmic-effect regime. Subsequent work was devoted to a more detailed analysis of the concept and its possible applications including a comparison with alternative propulsion and power generation methods [4]. It was shown that, depending on orbital and environmental conditions, EDTs can offer a considerably higher performance than chemical and electric propulsion not only for deorbiting operations (in which the absence of onboard power supplies makes the EDT system lighter) but also, although to a lesser extent, for orbit raising and drag compensation.

In addition to determining a preliminary design of a bare EDT for a specific space mission or space application, the mission analyst is generally interested in monitoring the behavior of the tether and its performance for the entire duration of the mission. Accurate knowledge of tether orbital and attitude dynamics evolution, instantaneous generated power, tether bowing, local temperature, etc., can be fundamental for the refinement of the EDT mission design and optimization as well as for the analysis of postmission data. These quantities can be estimated only if the current and bias profiles along the tether are known with sufficient accuracy.

The process of local bias and current profile estimation, i.e., the computation of the bias between the tether and the surrounding plasma at a specific point along the tether and the current flowing through it, is in general performed numerically and may be computationally expensive. Essentially, the estimation can be divided in two steps. First it is necessary to know the motional electric field projection along the tether line $\left(E_{t}\right)$ as well as the plasma density $\left(N_{e}\right)$ at a specific location. For earth-orbiting EDTs the computation of the plasma density is usually the heaviest numerical burden as it involves repeated access to a terrestrial ionosphere model such as the international reference ionosphere. Once these environmental quantities are known, the bias and current profile are determined by solving a two-point boundary-value problem consisting of two coupled differential equations, the local bias and current collection equations, the latter of which is nonlinear. Although a first integral for the system is available [3], the complete solution of the problem, with the exception of the limiting cases of zero ohmic effects and dominant ohmic effects with small load, is not available in simple analytical form and is usually handled numerically or by resorting to semi-analytical methods [5]. Clearly, in the context of EDT flight dynamics simulators, approximate analytical solutions would dramatically reduce the computation time needed and are therefore highly desirable. In addition, as the ionospheric density cannot be modeled with high-accuracy (primarily due to the influence of solar activity), errors in the bias and current profile by a few percent are totally acceptable.

In the present article, the computation of such a solution is dealt with by exploiting perturbation theory. The differential equations for the local bias and the current collection are written in nondimensional form in a manner which differs from the one utilized in the literature. With the present nondimensionalization, a parameter $\epsilon$ gauging the influence of ohmic effects appears directly in the equations so that the solution for small ohmic effects can be derived as an asymptotic expansion. When ohmic effects become large a different approach is used based on an extension of the exact analytical solution obtained for the case of zero load at the cathodic end.

The outline of the article is the following. First the bare EDT model used in this article is described and the main governing equations are introduced. The same equations are subsequently written in nondimensional form. Next, the case of zero ohmic effects is treated and an exact analytical solution is derived providing a physical interpretation of the parameter $\epsilon$. The case of small but not negligible ohmic effects is then solved with a perturbation method [6] including terms up to $\epsilon^{3}$. The case of large ohmic effects is dealt with in the following section for the case of zero load at the cathodic end, which admits an exact analytical solution, and for the general case, for which an approximate solution can be derived. Once an accurate and complete analytical solution is derived for the current and potential profile one can compute relevant quantities for the design and analysis of electrodynamic tether mission, i.e., resulting Lorentz force and torque, power generated and optimum load impedance for power generation. These quantities are then used as evaluation metrics to establish the accuracy of the analytical solution when compared with the exact numerically derived one and to establish the boundary between small and large ohmic effects. A comparison in terms of computational speed between the proposed analytical solution and the numerically-computed one is also performed.

\section{Tether Model and Governing Equations}

The model considered here consists of a bare EDT of length $L$ having uniform cross section of area $A$ and perimeter $p$ and made up with conductive material of density $\rho$ and conductivity $\sigma$. A plasma contactor (e.g. a hollow cathode) is inserted at the cathodic end ${ }^{\S}$ of the tether together with a load of impedance $Z_{\ell}$ placed just before the plasma contactor for power generation purposes (Fig. 1). The tether interacts with the ambient plasma characterized by electron density $N_{e}$ measured far enough from the tether line and local magnetic field intensity $B$, and moves with relative velocity $\Delta v$ with respect to the plasma. The quantities $N_{e}, B$ and $\Delta v$ are assumed constant along the tether. When electrical contact with the plasma is established electrons are collected along the (upper, in Fig. 1) positively biased segment resulting in a nonuniform current $I(x)$ flowing along the tether towards the cathodic contactor. In the general case in which the potential drop of the plasma contactor $\Delta V_{\mathrm{HC}}$ and/or the impedance $Z_{\ell}$ of the applied load is not zero the lower (in Fig. 1) portion of the tether will be negatively biased with respect to the plasma and will, unless insulated, collect ions. The current collection is here assumed to take place in the orbital motion limited (OML) regime [2,3]. A discussion on the limits of OML theory and the general case of EDT working beyond the OML regime will be presented in a later section.

\section{A. Main Equations}

Once the physical characteristics of the tether and the surrounding plasma environment are known the current and potential profile can 


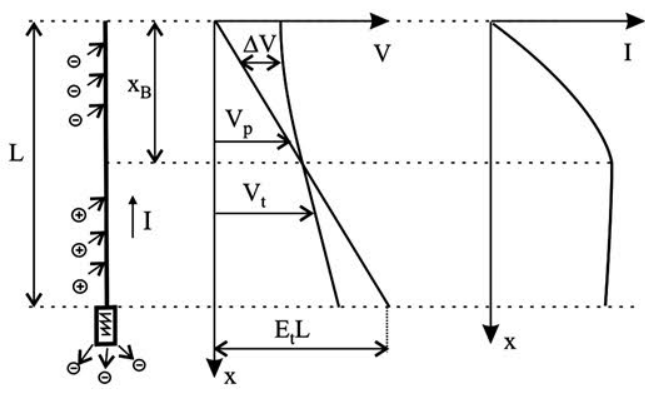

Fig. 1 Schematic of passive bare EDT with potential and current profiles. In this particular example we set $\xi_{B}=0.5, \epsilon=1.5$ and $\mu=1 / 43$.

be completely determined by the local bias equation and the current collection equation with appropriate boundary conditions. The first describes the potential difference $\Delta V=V_{t}-V_{p}$ between the tether and the surrounding plasma along the tether line $(0<x<L)$ and yields

$$
\frac{\mathrm{d}(\Delta V)}{\mathrm{d} x}=\frac{I(x)}{\sigma A}-E_{t}
$$

where $E_{t}$ is the magnitude of the motional electric field projected on the tether line. The boundary condition needed to solve Eq. (1) can be enforced using the identity

$$
\Delta V\left(x_{B}\right)=0
$$

with $x_{B}$ indicating the zero-bias abscissa.

The OML current collection equations [3] yield

$$
\begin{gathered}
\frac{\mathrm{d} I_{a}}{\mathrm{~d} x}=\frac{p}{\pi} q_{e} N_{e} \sqrt{\frac{2 q_{e}}{m_{e}} \Delta V} \\
\frac{\mathrm{d} I_{c}}{\mathrm{~d} x}=-\frac{p}{\pi} q_{e} N_{e} \sqrt{-\frac{2 q_{e}}{m_{i}} \Delta V,}
\end{gathered}
$$

where $N_{e}$ is the electron plasma density, $p$ is the tether cross-section perimeter, $q_{e}$ and $m_{e}$ are the electron charge and mass while $m_{i}$ can be taken as the mass of the most abundant ion species. Note that secondary-electron emission in the cathodic part is here assumed negligible. Here, and from now on, the subscripts $a$ and $c$ will refer to the anodic $\left(0<x<x_{B}\right)$ and cathodic $\left(x_{B}<x<1\right)$ section of the tether. Note that in case the cathodic segment is insulated the right member of Eq. (4) becomes zero.

The boundary conditions for the current collection equations yield

$$
\begin{gathered}
I_{a}(0)=0 \\
I_{a}\left(x_{B}\right)=I_{c}\left(x_{B}\right)
\end{gathered}
$$

Finally, we write the circuit equation at the cathodic end $(x=L)$ as

$$
\Delta V(L)=-\Delta V_{\mathrm{HC}}-Z_{\ell} I(L)
$$

where $\Delta V_{\mathrm{HC}}$ is the potential difference between the hollow cathode and the surrounding plasma." Equation (7) will be used to relate the load impedance $Z_{\ell}$ to the zero-bias length $x_{B}$.

\section{B. Dimensionless Equations}

We will now introduce the nondimensional abscissa, bias and current as follows:

\footnotetext{
${ }^{\top}$ As we are considering a completely passive system this potential difference is obtained at the expense of the electric-field-induced bias and must appear in the circuit equation.
}

$$
\xi=\frac{x}{L}, \quad \varphi=\frac{\Delta V}{E_{t} L}, \quad i=\frac{I}{I_{\mathrm{ch}}}
$$

where the characteristic current $I_{\text {ch }}$ is here taken as the maximum current which can be obtained along the tether in absence of ohmic losses and with both the load impedance and the potential drop at the cathode set to zero. This quantity will be computed later. The nondimensional form of Eqs. (1), (3), and (4) yields

$$
\begin{gathered}
\frac{\mathrm{d} \varphi}{\mathrm{d} \xi}=\epsilon i-1 \\
\frac{\mathrm{d} i_{a}}{\mathrm{~d} \xi}=\frac{3}{2} \alpha_{0} \sqrt{\varphi} \\
\frac{\mathrm{d} i_{c}}{\mathrm{~d} \xi}=-\frac{3}{2} \alpha_{0} \mu \sqrt{-\varphi}
\end{gathered}
$$

with boundary conditions

$$
\begin{gathered}
\varphi\left(\xi_{B}\right)=0 \\
i(0)=0 \\
i_{a}\left(\xi_{B}\right)=i_{c}\left(\xi_{B}\right)
\end{gathered}
$$

where the parameters $\epsilon, \alpha_{0}$ and $\mu$ are defined as

$$
\begin{gathered}
\epsilon=\frac{I_{\mathrm{ch}}}{\sigma A E_{t}} \\
\alpha_{0}=\frac{2}{3} \frac{q_{e} N_{e}}{I_{\mathrm{ch}}} \frac{p L^{3 / 2}}{\pi} \sqrt{\frac{2 q_{e} E_{t}}{m_{e}}} \\
\mu=\sqrt{\frac{m_{e}}{m_{i}}}
\end{gathered}
$$

with the latter known as ion mass ratio.

The parameter $0<\xi_{B}<1$ corresponds to the nondimensional length of the anodic segment, here defined as the segment whose potential difference with respect to the surrounding plasma is positive or zero, and will be treated as an independent variable for current control and optimization purposes. Once the value of the bias and the current at the cathode are known as well as the potential drop at the cathodic contactor the load impedance corresponding to a given value of $\xi_{B}$ can be determined through the dimensionless version of Eq. (7) which yields

$$
\varphi_{c}(1)=-v_{\mathrm{HC}}-i_{c}(1) \Omega
$$

where

$$
\Omega=\frac{I_{\mathrm{ch}} Z_{\ell}}{E_{t} L}, \quad v_{\mathrm{HC}}=\frac{\Delta V_{\mathrm{HC}}}{E_{t} L}
$$

are the nondimensional load impedance and the nondimensional potential drop at the cathodic plasma contactor, respectively.

\section{Case of Zero Ohmic Effects}

In this section we will address the idealized case in which ohmic effects are zero so that the achievable current in the tether is entirely determined by the collection impedance of the latter with respect to the plasma. This may also be called collection-limited regime. In this case an exact solution of the bias and current profile is possible, which allows one to introduce the fundamental parameters required for the solution of the general case. 


\section{A. Analytical Solution}

When $\epsilon=0$ an exact solution is easily found by first solving Eq. (8) with boundary condition [Eq. (11)] and, in cascade, by solving Eqs. (9) and (10) with boundary condition [Eqs. (12) and (13)] to obtain

$$
\begin{gathered}
\varphi_{0}(\xi)=\xi_{B}-\xi \\
i_{0 a}(\xi)=\alpha_{0}\left[\xi_{B}^{3 / 2}-\left(\xi_{B}-\xi\right)^{3 / 2}\right] \\
i_{0 c}(\xi)=\alpha_{0}\left[\xi_{B}^{3 / 2}-\mu\left(\xi-\xi_{B}\right)^{3 / 2}\right]
\end{gathered}
$$

In dimensional form the peak value of the tether current, $I_{B}^{0}$ reads

$$
I_{B}^{0}=\frac{2 p}{3 \pi} N_{e} \sqrt{\frac{2 E_{t}}{m_{e}} q_{e}^{3} L^{3}} \cdot \xi_{B}^{3 / 2}
$$

Once the control parameter $\xi_{B}$ is set the corresponding dimensionless load impedance (for zero ohmic effects) can be determined after substituting Eqs. (18) and (20) into Eq. (17) obtaining

$$
\Omega=\frac{1}{\alpha_{0}} \cdot \frac{1-\xi_{B}-v_{\mathrm{HC}}}{\xi_{B}^{3 / 2}-\mu\left(1-\xi_{B}\right)^{3 / 2}}
$$

When the load impedance is set to zero and in the ideal case in which the potential drop at the cathode is also zero $\xi_{B}=1$ and $I_{B}^{0}$ reaches its maximum value providing the characteristic current:

$$
I_{\mathrm{ch}}=\frac{2 p}{3 \pi} N_{e} \sqrt{\frac{2 E_{t}}{m_{e}} q_{e}^{3} L^{3}}
$$

By substituting Eq. (23) into Eq. (15) the constant $\alpha_{0}$ becomes unity and the bias and current profile simplify to

$$
\begin{gathered}
\varphi_{0}(\xi)=\xi_{B}-\xi \\
i_{0 a}(\xi)=\xi_{B}^{3 / 2}-\left(\xi_{B}-\xi\right)^{3 / 2} \\
i_{0 c}(\xi)=\xi_{B}^{3 / 2}-\mu\left(\xi-\xi_{B}\right)^{3 / 2}
\end{gathered}
$$

An interesting limit case occurs when the dimensionless load impedance is set to infinity, which is equivalent to having zero current at the cathodic end or, in other words, a system working without a cathodic plasma contactor. This is what is called floating EDT (see [7]). From Eq. (22) one can derive the corresponding zero-bias length**:

$$
\xi_{B}^{*}=\frac{\mu^{2 / 3}}{\mu^{2 / 3}+1} \cong \mu^{2 / 3}
$$

Note that if the cathodic segment is uninsulated $\xi_{B}^{*}$ becomes the lower bound, in place of zero, for the free parameter $\xi_{B}$.

\section{B. Parameter $\epsilon$}

The expression of $\epsilon$ given by Eq. (14) can also be simplified through Eq. (23) to yield

$$
\epsilon=\frac{2 p}{3 \pi} N_{e}\left(\frac{L}{\sigma A}\right) \sqrt{\frac{2 L q_{e}^{3}}{m_{e} E_{t}}}=\frac{Z_{t}}{Z_{c}}
$$

where

${ }^{* *}$ Note that secondary electron emission in the cathodic part is neglected
here.

$$
Z_{t}=\frac{L}{\sigma A}
$$

and

$$
Z_{c}=\frac{3 \pi}{2 p \sqrt{L}} \frac{\sqrt{E_{t}}}{N_{e}} \sqrt{\frac{m_{e}}{2 q_{e}^{3}}}
$$

are, respectively, the ohmic impedance of the conducting tether and the equivalent plasma contact impedance of the tether defined as

$$
Z_{c}=\frac{E_{t} L}{I_{\mathrm{ch}}}
$$

The parameter $\epsilon$ depends on the tether geometry $(p, L, A)$, tether conductivity $(\sigma)$ local plasma density $N_{e}$ and on the projected motional electric field $E_{t}$. The material of choice for current EDT application is aluminum, which thanks to its low density $(\rho \approx$ $\left.2600 \mathrm{~kg} / \mathrm{m}^{3}\right)$ and high conductivity $\left(\sigma \approx 3.5 \times 10^{7} \mathrm{ohm}^{-1} \cdot \mathrm{m}^{-1}\right)$, offers high performance in terms of thrust- and power-to-mass ratio. In addition, as aluminum has high malleability, a very thin tapelike cross section can be employed to further increase performance. Ultimately we can consider a thin (say $0.05 \mathrm{~mm}$ ) aluminum tape tether as our baseline design and examine the variability of the parameter $\epsilon$ due to tether length and orbit environment characteristics.

In Earth orbit the electron density $N_{e}$ undergoes both spatial and temporal variations. In the F-region of the ionosphere (160 to $1000 \mathrm{~km}$ altitude), the electron density reaches a maximum of $10^{11}$ to $10^{13} \mathrm{~m}^{-3}$ (depending on solar activity and taking into account the day-night cycle), mostly due to the contribution of atomic oxygen $(\mu \simeq 1 / 171)$ and decreases exponentially by almost two orders of magnitude up to $600-700 \mathrm{~km}$ altitude where the lighter hydrogen ions $(\mu \simeq 1 / 43$ ) become the dominant species. The projected motional electric field $E_{t}$ is in general a function of the tether position and velocity along the orbit as well as its attitude. On the other hand, if we consider circular equatorial orbits with the tether stabilized along the local vertical and we neglect the small magnetic field variations along the orbit due to tilt, offset and higher harmonics, $E_{t}$ becomes solely a function of the orbit radius $r$ so that, for a prograde $(-)$ and retrograde $(+)$ orbit, we have

$$
E_{t} \cong\left(\sqrt{\frac{\mu_{p}}{r}} \pm \omega_{p} r\right) \times \frac{B_{S} r_{p}^{3}}{r^{3}}
$$

where $\mu_{p}, r_{p}, \omega_{p}$ and $B_{S}$ are, respectively, the planet gravitational constant, the planet radius, the angular velocity of the planetcorotating plasmasphere and the planet surface magnetic field measured at the magnetic equator.

Figure 2 illustrates the variability of $\epsilon$ [Eq. (27)] considering Earth prograde circular equatorial orbits of different altitude and aluminum tape tethers with fixed $(10 \mathrm{~km})$ length and $(0.05 \mathrm{~mm})$ thickness. Note that for the general case of inclined orbits the motional electric field varies along the orbit in a complex manner where higher magnetic field harmonics may play an important role. An analysis of the range of variation of epsilon in such case, which clearly cannot be summarized with a simple plot, is beyond the scope of this article.

In Jupiter orbits, which are characterized by considerably lower electron density and higher electric field, the parameter $\epsilon$ is much smaller. Figure 3 plots the variability of $\epsilon$ for the same tether considered above and placed in equatorial prograde Jupiter orbits. A Divine-Garrett ionosphere model [8] has been employed. Ohmic effects are small around Jupiter but can be important in the vicinity of the Io torus or very close to Jupiter when relatively long tethers (say $>20 \mathrm{~km}$ ) are considered.

\section{Solution for Small Ohmic Effects}

In this section we will address the case in which the ohmic impedance of the conductive tether is small when compared with the equivalent plasma contact impedance of the bare tether. This case allows to address the solution of the bias and current collection 


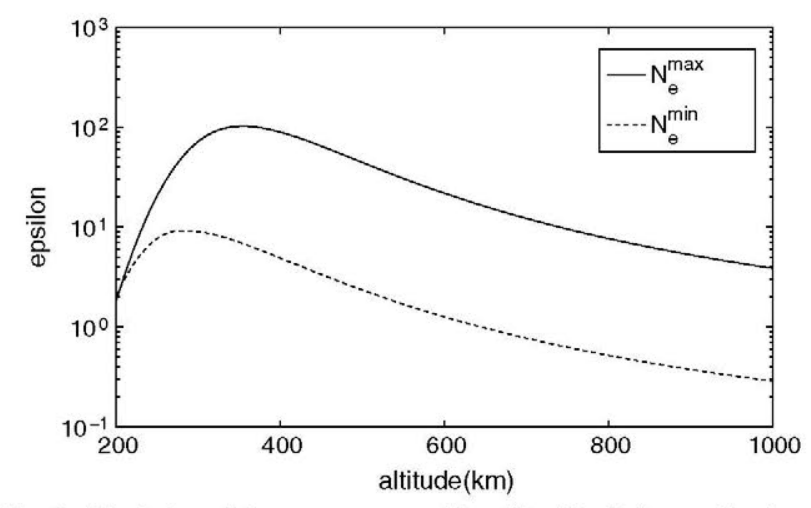

Fig. 2 Variation of the parameter $\epsilon$ with orbit altitude for an aluminum tape EDT in equatorial prograde circular orbit around the Earth. Tape length and thickness are set to $10 \mathrm{~km}$ and $0.05 \mathrm{~mm}$, respectively. $N_{e}^{\max }\left(N_{e}^{\min }\right)$ refers to the electron density computed at daylight (nighttime) during maximum (minimum) solar activity across the 11-year solar cycle. The plot scales with tether length and thickness as $L^{3 / 2}$ and $h^{-1}$, respectively.

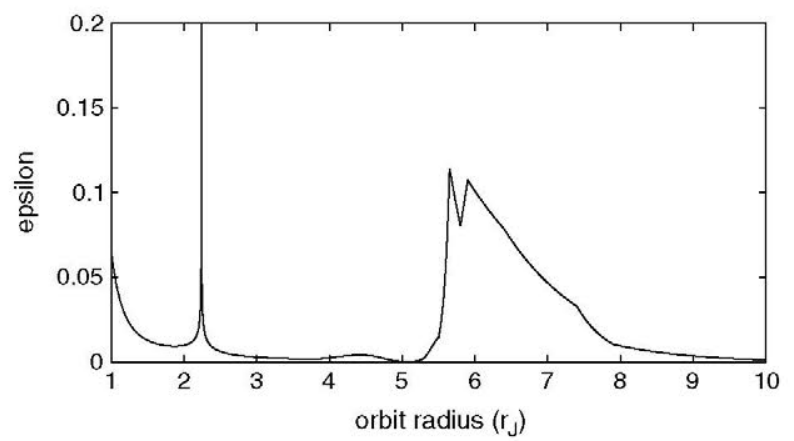

Fig. 3 Variation of $\epsilon$ with orbital radius for an aluminum tape EDT in equatorial prograde circular orbit around Jupiter. Tape and thickness are set to $10 \mathrm{~km}$ and $0.05 \mathrm{~mm}$, respectively. The parameter $\epsilon$ scales with tether length and thickness as $L^{3 / 2}$ and $h^{-1}$, respectively. Note that epsilon goes to infinity at $\sim 2.2 r_{J}$ where the electric field is zero (Jovesynchronous altitude) and approaches its maximum value close to the orbit of Io due to the high plasma density of the Io torus.

equations with the aid of an asymptotic expansion. It will be seen later that the solution obtained with this method provides a relative error smaller than $5 \%$ on the average current as long as the parameter epsilon obeys $\epsilon \lesssim 8 /\left(5 \xi_{B}^{3 / 2}\right)$ while beyond such limit a different approach will provide better accuracy (see Fig. 4 ). We will therefore refer to the small ohmic effects regime when

$$
0<\epsilon<\frac{8}{5 \xi_{B}^{3 / 2}}
$$

and to the dominant ohmic effects regime when

$$
\epsilon>\frac{8}{5 \xi_{B}^{3 / 2}}
$$

If condition Eq. (32) is verified the current and potential functions can be conveniently replaced with the expansion

$$
\varphi^{S}\left(\xi_{B}, \xi, \epsilon\right)=\varphi_{0}\left(\xi, \xi_{B}\right)+\epsilon \varphi_{1}\left(\xi, \xi_{B}\right)+\epsilon^{2} \varphi_{2}\left(\xi, \xi_{B}\right)+\epsilon^{3} \varphi_{3}\left(\xi, \xi_{B}\right)
$$

$$
i^{S}\left(\xi_{B}, \xi, \epsilon\right)=i_{0}\left(\xi, \xi_{B}\right)+\epsilon i_{1}\left(\xi, \xi_{B}\right)+\epsilon^{2} i_{2}\left(\xi, \xi_{B}\right)
$$

After substituting the previous equations into Eqs. (8-10) and expanding in Taylor series and collecting coefficients of like power of $\epsilon$ we obtain

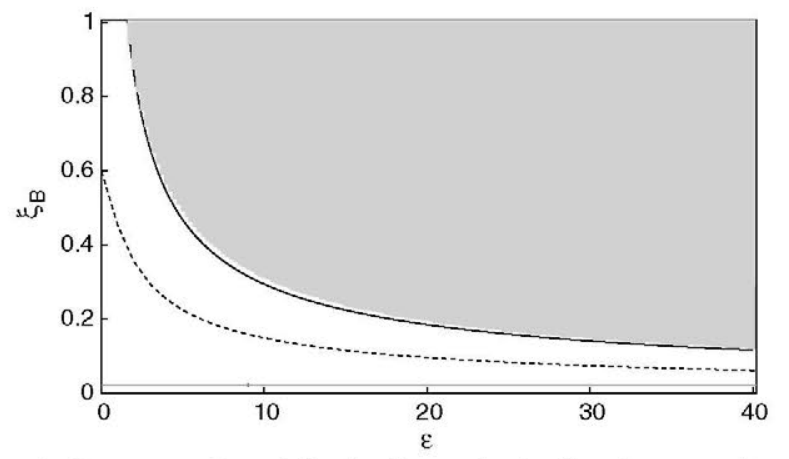

Fig. 4 Representation of the dominant-ohmic-effect (gray area) and small-ohmic-effect region in the $\left(\epsilon, \xi_{B}\right)$ parameter space for a passive EDT. The boundary between the two regions is well represented by the function $\xi_{B}=(8 / 5 \epsilon)^{2 / 3}$ (dark solid line). The dark dash-line represents optimum power generation conditions for the case of $\mu=0$ (insulated cathodic segment). Finally the gray solid line represents the floating condition $\left(\xi_{B} \approx \mu^{2 / 3}\right)$ for a fully bare tether considering $\mu=1 / 43$ (dominant hydrogen ions) and neglecting secondary emissions. Note that for a passive noninsulated EDT $\xi_{B}$ cannot go below the floating-condition value.

$$
\begin{gathered}
\frac{\mathrm{d} \varphi_{0}}{\mathrm{~d} \xi}+1+\epsilon\left(\frac{\mathrm{d} \varphi_{1}}{\mathrm{~d} \xi}-i_{0}\right)+\epsilon^{2}\left(\frac{\mathrm{d} \varphi_{2}}{\mathrm{~d} \xi}-i_{1}\right) \\
+\epsilon^{3}\left(\frac{\mathrm{d} \varphi_{3}}{\mathrm{~d} \xi}-i_{2}\right)+o\left(\epsilon^{3}\right)=0 \\
\frac{\mathrm{d} i_{0 a}}{\mathrm{~d} \xi}-\frac{3}{2} \sqrt{\varphi_{0 a}}+\epsilon\left(\frac{\mathrm{d} i_{1 a}}{\mathrm{~d} \xi}-\frac{3}{4} \frac{\varphi_{1 a}}{\sqrt{\varphi_{0 a}}}\right) \\
+\epsilon^{2}\left(\frac{\mathrm{d} i_{2 a}}{\mathrm{~d} \xi}-\frac{3}{4} \frac{\varphi_{2 a}}{\sqrt{\varphi_{0 a}}}+\frac{3}{16} \frac{\varphi_{1 a}^{2}}{\varphi_{0 a}^{3 / 2}}\right)+o\left(\epsilon^{2}\right)=0 \\
\mathrm{~d} \xi \\
+\frac{3}{2} \mu \sqrt{-\varphi_{0 c}}+\epsilon\left(\frac{\mathrm{d} i_{1 c}}{\mathrm{~d} \xi}-\frac{3}{4} \mu \frac{\varphi_{1 c}}{\sqrt{-\varphi_{0 c}}}\right) \\
+\epsilon^{2}\left(\frac{\mathrm{d} i_{2 c}}{\mathrm{~d} \xi}+\frac{3}{4} \mu \frac{\varphi_{2 c}}{\sqrt{-\varphi_{0 c}}}-\frac{3}{16} \mu \frac{\varphi_{1 c}^{2}}{\left(-\varphi_{0 c}\right)^{3 / 2}}\right)+o\left(\epsilon^{2}\right)=0
\end{gathered}
$$

Note that the local bias equation has been expanded to one additional order to gain better accuracy as it will be proved by comparison with the numerical solution. Conversely, higher order components for the current profile do not help in gaining better accuracy and may deteriorate the convergence as $\epsilon$ increases.

The zeroth order solution ( $\epsilon=0)$, corresponding to the zero ohmic effects case, is given by Eqs. (24-26). By substituting the latter into Eq. (36) the first-order differential equations for the potential read

$$
\begin{aligned}
\frac{\mathrm{d} \varphi_{1 a}}{\mathrm{~d} \xi} & =\xi_{B}^{3 / 2}-\left(\xi_{B}-\xi\right)^{3 / 2} \\
\frac{\mathrm{d} \varphi_{1 c}}{\mathrm{~d} \xi} & =\xi_{B}^{3 / 2}-\mu\left(\xi-\xi_{B}\right)^{3 / 2}
\end{aligned}
$$

which solved with boundary condition $\varphi_{1}\left(\xi_{B}\right)=0$ yield

$$
\begin{gathered}
\varphi_{1 a}\left(\xi_{B}, \xi\right)=\left(\xi_{B}-\xi\right)\left[\frac{2}{5}\left(\xi_{B}-\xi\right)^{3 / 2}-\xi_{B}^{3 / 2}\right] \\
\varphi_{1 c}\left(\xi_{B}, \xi\right)=\left(\xi-\xi_{B}\right)\left[-\frac{2}{5} \mu\left(\xi-\xi_{B}\right)^{3 / 2}+\xi_{B}^{3 / 2}\right]
\end{gathered}
$$

After substituting Eqs. (25), (26), (41), and (42) into Eqs. (37) and (38) the first-order differential equations for the current read 


$$
\begin{gathered}
\frac{\mathrm{d} i_{1 a}}{\mathrm{~d} \xi}=\frac{3}{10}\left(\xi_{B}-\xi\right)^{2}-\frac{3}{4} \xi_{B}^{3 / 2}\left(\xi_{B}-\xi\right)^{1 / 2} \\
\frac{\mathrm{d} i_{1 c}}{\mathrm{~d} \xi}=-\frac{3}{10} \mu^{2}\left(\xi-\xi_{B}\right)^{2}+\frac{3}{4} \mu \xi_{B}^{3 / 2}\left(\xi-\xi_{B}\right)^{1 / 2}
\end{gathered}
$$

which solved with boundary conditions $i_{1 a}(0)=0$ and $i_{2 c}\left(\xi_{B}\right)=$ $i_{2 a}\left(\xi_{B}\right)$ yield

$$
\begin{aligned}
& i_{1 a}\left(\xi_{B}, \xi\right)=-\frac{2}{5} \xi_{B}^{3}+\frac{1}{2} \xi_{B}^{3 / 2}\left(\xi_{B}-\xi\right)^{3 / 2}-\frac{1}{10}\left(\xi_{B}-\xi\right)^{3} \\
& i_{1 c}\left(\xi_{B}, \xi\right)=-\frac{2}{5} \xi_{B}^{3}+\frac{\mu}{2} \xi_{B}^{3 / 2}\left(\xi-\xi_{B}\right)^{3 / 2}-\frac{\mu^{2}}{10}\left(\xi-\xi_{B}\right)^{3}
\end{aligned}
$$

The procedure can be repeated for the second-order case obtaining

$$
\varphi_{2 a}\left(\xi_{B}, \xi\right)=\left(\xi_{B}-\xi\right)\left[\frac{2}{5} \xi_{B}^{3}-\frac{1}{5} \xi_{B}^{3 / 2}\left(\xi_{B}-\xi\right)^{3 / 2}+\frac{1}{40}\left(\xi_{B}-\xi\right)^{3}\right]
$$

$$
\varphi_{2 c}\left(\xi_{B}, \xi\right)=\left(\xi-\xi_{B}\right)\left[-\frac{2}{5} \xi_{B}^{3}+\frac{\mu}{5} \xi_{B}^{3 / 2}\left(\xi-\xi_{B}\right)^{3 / 2}-\frac{\mu^{2}}{40}\left(\xi-\xi_{B}\right)^{3}\right]
$$

and

$$
\begin{aligned}
& i_{2 a}\left(\xi_{B}, \xi\right)=\frac{1}{400}\left[29 \xi_{B}^{9 / 2}-30 \xi_{B}^{3}\left(\xi_{B}-\xi\right)^{3 / 2}+\left(\xi_{B}-\xi\right)^{9 / 2}\right] \\
& i_{2 c}\left(\xi_{B}, \xi\right)=\frac{1}{400}\left[29 \xi_{B}^{9 / 2}-30 \mu \xi_{B}^{3}\left(\xi-\xi_{B}\right)^{3 / 2}+\mu^{3}\left(\xi-\xi_{B}\right)^{9 / 2}\right]
\end{aligned}
$$

The third-order solution is computed only for the potential and yields

$$
\begin{gathered}
\varphi_{3 a}\left(\xi_{B}, \xi\right)=\frac{\xi_{B}-\xi}{4400}\left[-319 \xi_{B}^{9 / 2}+132 \xi_{B}^{3}\left(\xi_{B}-\xi\right)^{3 / 2}-2\left(\xi_{B}-\xi\right)^{9 / 2}\right] \\
\varphi_{3 c}\left(\xi_{B}, \xi\right)=\frac{\xi-\xi_{B}}{4400}\left[319 \xi_{B}^{9 / 2}-132 \mu \xi_{B}^{3}\left(\xi-\xi_{B}\right)^{3 / 2}\right. \\
\left.+2 \mu^{3}\left(\xi-\xi_{B}\right)^{9 / 2}\right]
\end{gathered}
$$

Finally, our second- and third-order approximation for the potential and current profile can be written as

$$
\begin{aligned}
& \varphi_{a}^{S}\left(\xi_{B}, \xi\right)=\left(\xi_{B}-\xi\right)\left\{1+\epsilon\left[\frac{2}{5}\left(\xi_{B}-\xi\right)^{3 / 2}-\xi_{B}^{3 / 2}\right]\right. \\
& +\epsilon^{2}\left[\frac{2}{5} \xi_{B}^{3}-\frac{1}{5} \xi_{B}^{3 / 2}\left(\xi_{B}-\xi\right)^{3 / 2}+\frac{1}{40}\left(\xi_{B}-\xi\right)^{3}\right] \\
& \left.+\frac{\epsilon^{3}}{4400}\left[-319 \xi_{B}^{9 / 2}+132 \xi_{B}^{3}\left(\xi_{B}-\xi\right)^{3 / 2}-2\left(\xi_{B}-\xi\right)^{9 / 2}\right]\right\} \\
& \varphi_{c}^{S}\left(\xi_{B}, \xi\right)=\left(\xi-\xi_{B}\right)\left\{-1+\epsilon\left[-\frac{2}{5} \mu\left(\xi-\xi_{B}\right)^{3 / 2}+\xi_{B}^{3 / 2}\right]\right. \\
& +\epsilon^{2}\left[-\frac{2}{5} \xi_{B}^{3}+\frac{\mu}{5} \xi_{B}^{3 / 2}\left(\xi-\xi_{B}\right)^{3 / 2}-\frac{\mu^{2}}{40}\left(\xi-\xi_{B}\right)^{3}\right] \\
& \left.+\frac{\epsilon^{3}}{4400}\left[319 \xi_{B}^{9 / 2}-132 \mu \xi_{B}^{3}\left(\xi-\xi_{B}\right)^{3 / 2}+2 \mu^{3}\left(\xi-\xi_{B}\right)^{9 / 2}\right]\right\} \\
& i_{a}^{S}\left(\xi_{B}, \xi\right)=\xi_{B}^{3 / 2}-\left(\xi_{B}-\xi\right)^{3 / 2}+\frac{\epsilon}{10}\left[-4 \xi_{B}^{3}+5 \xi_{B}^{3 / 2}\left(\xi_{B}-\xi\right)^{3 / 2}\right. \\
& \quad-\left(\xi_{B}-\xi^{3 / 2}\right]+\frac{\epsilon^{2}}{400}\left[29 \xi_{B}^{9 / 2}-30 \xi_{B}^{3}\left(\xi_{B}-\xi\right)^{3 / 2}+\left(\xi_{B}-\xi\right)^{9 / 2}\right]
\end{aligned}
$$

$$
\begin{aligned}
& i_{c}^{S}\left(\xi_{B}, \xi\right)=\xi_{B}^{3 / 2}-\mu\left(\xi-\xi_{B}\right)^{3 / 2} \\
& \quad+\frac{\epsilon}{10}\left[-4 \xi_{B}^{3}+5 \mu \xi_{B}^{3 / 2}\left(\xi-\xi_{B}\right)^{3 / 2}-\mu^{2}\left(\xi-\xi_{B}\right)^{3}\right] \\
& \quad+\frac{\epsilon^{2}}{400}\left[29 \xi_{B}^{9 / 2}-30 \mu \xi_{B}^{3}\left(\xi-\xi_{B}\right)^{3 / 2}+\mu^{3}\left(\xi-\xi_{B}\right)^{9 / 2}\right]
\end{aligned}
$$

At this point one can compute the value of the interposed load impedance necessary to obtain a given value of $\xi_{B}$. This can be done by solving Eq. (17) for $\Omega$ :

$$
\Omega=\frac{-\varphi_{c}^{S}(1)-v_{\mathrm{HC}}}{i_{c}^{S}(1)}
$$

and inserting the expressions of $\varphi_{c}^{S}(1)$ and $i_{c}^{S}(1)$ given in Eqs. (54) and (56).

For the case in which the value of the interposed load impedance is used as an independent variable (in place of $\xi_{B}$ ), the algebraic Eq. (57) has to be solved numerically. Spurious solutions can, however, appear in this case and the reader should carefully check the physical validity of the results obtained. The particular case in which $\Omega=0$ and $v_{\mathrm{HC}} \neq 0$ has high relevance in EDT applications and is dealt with in a following section.

\section{Solution for Large Ohmic Effects}

In this section we will derive an analytical solution when the parameters $\epsilon$ and $\xi_{B}$ satisfy the inequality Eq. (33) meaning the EDT works in the dominant ohmic effects regime. In such case, the solution given by Eqs. (53-56) provides unacceptably poor accuracy and quickly diverges for high enough values of $\epsilon$ and $\xi_{B}$.

From a physical point of view, as $\epsilon$ grows due to increasing ohmic effects the maximum current along the tether approaches the critical value:

$$
i_{\mathrm{sc}}=1 / \epsilon
$$

called short-circuit current, which brings to zero the derivative of the potential profile [Eq. (8)]. Later on we will show that, as it is known from the literature [9], when no load is applied at the cathodic end, and in the ideal case in which the potential drop at the cathode is zero, such critical value is reached for $\epsilon=4$ at the very end of the tether line. Under the same assumptions, if $\epsilon$ is further increased $i_{\mathrm{sc}}$ is reached before the end of the tether so that the last tether segment will not collect electrons neither ions. Conversely, for the general case in which a nonzero load is inserted at the cathodic end, $\dot{i}_{\mathrm{sc}}$ can only be reached if the load impedance does not exceed a critical value. In the first case an exact analytical solution will be found, which will then be used as the basis of an accurate approximate solution for the general case.

\section{A. Case of Zero Load $\left(Z_{\ell}=\mathbf{0}\right)$}

Let us first consider the case in which no load is applied at the end of the tether and the plasma contactor potential drop $v_{\mathrm{HC}}$ is negligible. Under these assumptions, let us then suppose that, once a value of $\epsilon$ is fixed, there exists a point $\hat{\xi}$ along the tether such that

$$
i(\hat{\xi})=i_{\mathrm{sc}}
$$

From Eq. (8) this implies

$$
\frac{\mathrm{d} \varphi}{\mathrm{d} \xi}(\hat{\xi})=0
$$

We will now show that if we enforce boundary condition Eq. (17), which in this case reduces to $\varphi(1)=0$, both the potential and its derivative must necessarily be zero along the tether branch extending from $\xi=\hat{\xi}$ to the end of the tether:

$$
\varphi(\hat{\xi}<\xi<1)=0
$$




$$
\frac{\mathrm{d} \varphi}{\mathrm{d} \xi}(\hat{\xi}<\xi<1)=0
$$

This can be easily demonstrated by extracting the second derivative of the bias profile from Eqs. (8-10):

$$
\begin{gathered}
\frac{\mathrm{d}^{2} \varphi}{\mathrm{d} \xi^{2}}=\frac{3}{2} \epsilon \sqrt{\varphi} \quad \varphi>0 \\
\frac{\mathrm{d}^{2} \varphi}{\mathrm{d} \xi^{2}}=-\frac{3 \mu}{2} \epsilon \sqrt{-\varphi} \quad \varphi<0
\end{gathered}
$$

If it was $\varphi(\hat{\xi})>0$ the bias curve would remain convex in the interval $[\hat{\xi} 1]$ with first derivative increasing from zero to a positive value so that the bias $\varphi(1)$ ends up positive hence violating the cathode boundary condition $\varphi(1)=0$. Likewise it cannot be $\varphi(\hat{\xi})<$ 0 because in this case the bias curve would remain concave throughout the whole interval $\left[\begin{array}{ll}0 & 1\end{array}\right]$ hence ending up negative at the cathodic end. This means that $\varphi$ must be zero at $\xi=\hat{\xi}$ and, because $\varphi(\xi)$ and $\mathrm{d} \varphi / \mathrm{d} \xi$ are continuous functions, also at any point between $\hat{\xi}$ and 1 eventually leading to conditions Eqs. (61) and (62).

Condition Eq. (61) is in itself a boundary condition, which can be exploited to provide an exact analytical solution of the current and potential profile.

By eliminating the independent variable $\xi$ from Eqs. (8) and (9) one obtains

$$
\frac{3}{2} \sqrt{\varphi} \mathrm{d} \varphi=(\varepsilon i-1) \mathrm{d} i
$$

which can be integrated to provide

$$
\varphi^{3 / 2}=\frac{1}{2}\left(\varepsilon i^{2}-2 i\right)+\varphi_{0}^{3 / 2}
$$

By imposing boundary condition Eq. (61) at $\xi=\hat{\xi}$ Eq. (81) yields

$$
\varphi=\frac{1}{(2 \varepsilon)^{2 / 3}}(1-\varepsilon i)^{4 / 3}
$$

Finally, by substituting Eq. (67) into Eq. (9) and solving for $i$ and $\varphi$ we obtain the solution for the current and potential profile:

$$
\begin{gathered}
i(\xi)=\frac{1}{\varepsilon}\left[1-\left(1-\frac{\xi}{\hat{\xi}}\right)^{3}\right] \quad ; 0<\xi<\hat{\xi} \\
i(\xi)=\frac{1}{\varepsilon} \quad ; \hat{\xi}<\xi<1 \\
\varphi(\xi)=\frac{(\hat{\xi}-\xi)^{4}}{4 \hat{\xi}^{3}} \quad ; 0<\xi<\hat{\xi} \\
\varphi(\xi)=0 \quad ; \hat{\xi}<\xi<1
\end{gathered}
$$

where:

$$
\hat{\xi}=\left(\frac{4}{\epsilon}\right)^{2 / 3}
$$

Equation (72) shows that the point $\hat{\xi}$ at which the current reaches the short-circuit limit $i_{\mathrm{sc}}$ is physically located inside the tether as long as $\epsilon \geqslant 4$. In particular $\hat{\xi}$ is located at the end of the tether when $\epsilon=4$, while further increasing $\epsilon$ shifts $\hat{\xi}$ inward from the cathodic end in fact shrinking the tether anodic segment and preventing the remainder of the tether from collecting any charge.

This means that for $\epsilon \geqslant 4$ the solution given by Eqs. $(68-72)$ is, under the validity of the current model, exact. On the other hand, as it will be shown later, the same equations retain a very good accuracy (i.e. a relative error of less than $3 \%$ on the maximum current) also in the range $1.6<\epsilon<4$. This fact is important because it allows one to both extend the solution until the region of validity of the previously developed asymptotic solution and to find a high-accuracy solution for the case of dominant ohmic effects and nonzero load.

\section{B. General Case $\left(Z_{\ell} \neq 0\right)$}

Let us now consider the case in which a load is applied at the end of the tether and/or the potential drop at the cathode is nonnegligible. First we will look for a possible exact solution of the governing differential equations. Following the same reasoning as in the previous section we see that if the short-circuit current condition is reached in $\hat{\xi}$ then we have

$$
\begin{gathered}
\varphi(\hat{\xi})=0 \\
\frac{\mathrm{d} \varphi}{\mathrm{d} \xi}(\hat{\xi})=0
\end{gathered}
$$

As far as the anodic segment is concerned the (exact) solution must be the same as the zero load case since the boundary conditions have not changed:

$$
\begin{gathered}
i_{a}^{L}(\xi)=\frac{1}{\varepsilon}\left[1-\left(1-\frac{\xi}{\hat{\xi}}\right)^{3}\right] \quad ; 0<\xi<\hat{\xi} \\
\varphi_{a}^{L}(\xi)=\frac{(\hat{\xi}-\xi)^{4}}{4 \hat{\xi}^{3}} \quad ; 0<\xi<\hat{\xi}
\end{gathered}
$$

Now, after reminding that the parameter $\xi_{B}$ represent the nondimensional length of the tether segment whose potential difference with respect to the surrounding plasma is positive or zero, we assume $\xi_{B} \geqslant \hat{\xi}$ and look for an exact solution for the remainder of the tether with the following structure:

$$
\begin{gathered}
i_{c}^{L}\left(\xi_{B} \geqslant \hat{\xi}\right)= \begin{cases}1 / \epsilon & \hat{\xi}<\xi<\xi_{B} \\
f(\xi)<1 / \epsilon & \xi_{B}<\xi<1\end{cases} \\
\varphi_{c}^{L}\left(\xi_{B} \geqslant \hat{\xi}\right)= \begin{cases}0 & \hat{\xi}<\xi<\xi_{B} \\
g(\xi)<0 & \xi_{B}<\xi<1\end{cases}
\end{gathered}
$$

where the parameter $\xi_{B}$ satisfies

$$
\xi_{B} \geqslant \hat{\xi}
$$

By eliminating the independent variable $\xi$ from Eqs. (8) and (10) one obtains

$$
-\frac{3}{2} \mu \sqrt{-\varphi_{c}} \mathrm{~d} \varphi_{c}=\left(\varepsilon i_{c}-1\right) \mathrm{d} i_{c}
$$

which can be integrated to provide

$$
-\mu\left(-\varphi_{c}\right)^{3 / 2}=\frac{i_{c}}{2}\left(\varepsilon i_{c}-2\right)+\text { const }
$$

After substituting Eq. (10) into Eq. (83) and imposing boundary condition $\varphi\left(\xi_{B}\right)=0$ we finally obtain the exact solution:

$$
\begin{gathered}
g(\xi)=-\frac{\mu^{2} \epsilon^{2}}{64}\left(\xi-\xi_{B}\right)^{4} \\
f(\xi)=\frac{1}{\epsilon}-\frac{\mu^{2} \epsilon}{16}\left(\xi-\xi_{B}\right)^{3}
\end{gathered}
$$

From Eq. (17) one can derive the corresponding value of the nondimensional load impedance: 


$$
\Omega\left(\xi_{B} \geqslant \hat{\xi}\right)=\frac{\mu^{2} \epsilon^{3}\left(1-\xi_{B}\right)^{4}-64 \epsilon v_{\mathrm{HC}}}{64-4 \mu^{2} \epsilon^{2}\left(1-\xi_{B}\right)^{3}}
$$

which must be positive. Disregarding the case in which $\hat{\xi}<\mu^{2 / 3}$ (which corresponds to an unreasonably large $\epsilon$ ) the denominator in Eq. (86) is always positive which has to be true for the numerator as well. The latter condition provides an upper bound for $\xi_{B}$ whose range of variability, in order for the present solution to be valid, is finally

$$
\hat{\xi}<\xi_{B}<1-\frac{4 v_{H \mathrm{C}}^{1 / 4}}{\sqrt{\mu \epsilon}}
$$

Equation (84) can be solved numerically when a nonzero impedance $\Omega$ is used as independent variable in place of $\xi_{B}$. Note that in such case the maximum value of $\Omega$ for the present solution to be valid is

$$
\Omega_{\max }=\Omega\left(\xi_{B}=\hat{\xi}\right)=\frac{\mu^{2} \epsilon^{3}(1-\hat{\xi})^{4}-64 \epsilon v_{\mathrm{HC}}}{64-4 \mu^{2} \epsilon^{2}(1-\hat{\xi})^{3}}
$$

Note that when $\Omega=0$ but $v_{\mathrm{HC}} \neq 0$ Eq. (84) provides the closedform solution $\xi_{B}(\Omega=0)=1-\frac{4 v_{\text {HC }}^{1 / 4}}{\sqrt{\mu \epsilon}}$.

Finally the maximum nondimensional bias of the plasma contactor, in order for the present solution to be valid is obtained from Eq. (85) as

$$
v_{\mathrm{HC}, \max }=\frac{\mu^{2}}{256} \frac{\left(\epsilon^{2 / 3}-2^{4 / 3}\right)^{4}}{\epsilon^{2 / 3}}
$$

Unfortunately, the last condition is difficult to meet even with hollow cathodes exhibiting very small potential drop (a few tens of volts) especially if $\epsilon$ is not that large, which makes the derived solution less interesting for real EDT applications.

For the case in which $\xi_{B}<\hat{\xi}$ the short-circuit current cannot be reached, which prevents us from finding an exact analytical solution. While for values of $\xi_{B}$ which are close to $\hat{\xi}$ Eqs. (75), (76), (82), and (83) could still be used with sufficient accuracy, as $\xi_{B}$ is further decreased the error quickly becomes unacceptably large and the formulation described in the following is required.

As far as the anodic part is concerned $\left(0<\xi<\xi_{B}\right)$ we employ the profiles (in this case no longer exact) derived for the zero load case:

$$
\begin{gathered}
i_{a}^{L}\left(\xi_{B}<\hat{\xi}\right)=\frac{1}{\varepsilon}\left[1-\left(\frac{\hat{\xi}-\xi}{\hat{\xi}}\right)^{3}\right] \\
\varphi_{a}^{L}\left(\xi_{B}<\hat{\xi}\right)=\frac{(\hat{\xi}-\xi)^{4}}{4 \hat{\xi}^{3}}
\end{gathered}
$$

To derive the profiles for the cathodic part we introduce the following expansion based on the smallness of the parameter $\mu$ :

$$
\begin{gathered}
\varphi_{c}^{L}\left(\xi_{B}<\hat{\xi}\right)=\varphi_{0 c}(\xi)+\mu \varphi_{1 c}(\xi)+\mu^{2} \varphi_{2 c}(\xi)+\mathcal{O}\left(\mu^{3}\right) \\
i_{c}^{L}\left(\xi_{B}<\hat{\xi}\right)=i_{0 c}(\xi)+\mu i_{1 c}(\xi)+\mu^{2} i_{2 c}(\xi)+\mathcal{O}\left(\mu^{3}\right)
\end{gathered}
$$

By substituting into Eqs. (8) and (10) and expanding in Taylor series we obtain

$$
\begin{array}{r}
\frac{\mathrm{d} \varphi_{0 c}}{\mathrm{~d} \xi}+1-\epsilon i_{0 c}+\mu\left(\frac{\mathrm{d} \varphi_{1 c}}{\mathrm{~d} \xi}-\epsilon i_{1 c}\right) \\
+\mu^{2}\left(\frac{\mathrm{d} \varphi_{2 c}}{\mathrm{~d} \xi}-\epsilon i_{2 c}\right)+\mathcal{O}\left(\mu^{2}\right)=0
\end{array}
$$

$$
\begin{aligned}
& \frac{\mathrm{d} i_{0 c}}{\mathrm{~d} \xi}+\mu\left(\frac{\mathrm{d} i_{1 c}}{\mathrm{~d} \xi}-\frac{3}{2} \sqrt{-\varphi_{0 c}}\right) \\
& +\mu^{2}\left(\frac{\mathrm{d} i_{2 c}}{\mathrm{~d} \xi}-\frac{3}{4} \frac{\varphi_{1 c}}{\sqrt{-\varphi_{0 c}}}\right)+\mathcal{O}\left(\mu^{2}\right)=0
\end{aligned}
$$

Equations (92) and (93) can be solved in cascade to eventually provide a second-order approximation for the potential and current profile as

$$
\begin{gathered}
\left.\varphi_{c}^{L}\left(\xi_{B}<\hat{\xi}\right)\right)=-\frac{\left(\xi-\xi_{B}\right)\left(\hat{\xi}-\xi_{B}\right)^{3}}{\hat{\xi}^{3}}-\frac{2 \mu \epsilon}{5} \frac{\left(\xi-\xi_{B}\right)^{3 / 2}\left(\hat{\xi}-\xi_{B}\right)^{3 / 2}}{\hat{\xi}^{3 / 2}} \\
-\frac{\mu^{2} \epsilon^{2}}{40}\left(\xi-\xi_{B}\right)^{4}+\mathcal{O}\left(\mu^{2}\right) \\
\left.i_{c}^{L}\left(\xi_{B}<\hat{\xi}\right)\right)=\frac{1}{\varepsilon}\left[1-\frac{\left(\hat{\xi}-\xi_{B}\right)^{3}}{\hat{\xi}^{3}}\right]-\mu \epsilon\left(\xi-\xi_{B}\right)^{3 / 2}\left(\hat{\xi}-\xi_{B}\right)^{3 / 2} \\
-\frac{\mu^{2} \epsilon}{10}\left(\xi-\xi_{B}\right)^{3}+\mathcal{O}\left(\mu^{2}\right)
\end{gathered}
$$

The value of the nondimensional load impedance $\Omega$ to be applied in order to obtain a given value of $\xi_{B}$ can be again determined directly from Eq. (17) where the expressions for $\varphi_{c}(1)$ and $i_{c}(1)$ are the ones just derived.

\section{Influence of Plasma Contactor Potential Drop}

Although so far we have always considered $\xi_{B}$ as a known independent variable (in place of the nondimensional load impedance $\Omega$ ), for the case in which no power generation is performed but, instead, maximum current is desired, the load impedance is set to zero and $\xi_{B}$ becomes a function of the sole cathodic contactor potential drop $v_{\mathrm{HC}}$. As the latter is, in general, a given quantity, which depends on the particular cathodic device employed, it has to become the new independent variable for this particular case. In the following we present a method to compute $\xi_{B}$ after setting $\Omega=0$ and given a nonnegligible plasma contactor potential drop $v_{\mathrm{HC}}$.

When ohmic effects are small $\xi_{B}$ can be derived, for small enough values of $\epsilon$, with the help of the asymptotic expansion:

$$
\xi_{B}^{S}=\xi_{B 0}+\epsilon \xi_{B 1}+\epsilon^{2} \xi_{B 1}+\mathcal{O}(\epsilon)
$$

After substituting Eq. (96) into Eq. (57), setting $\Omega=0$, and solving for each term we obtain

$$
\begin{gathered}
\xi_{B 0}=1-v_{\mathrm{HC}} \\
\xi_{B 1}=-v_{\mathrm{HC}}\left(1-v_{\mathrm{HC}}\right)^{3 / 2}+\frac{2}{5} \mu v_{\mathrm{HC}}^{5 / 2} \\
\xi_{B 2}=-\frac{3}{10} v_{\mathrm{HC}}\left(1-v_{\mathrm{HC}}\right)^{2}\left(2-7 v_{\mathrm{HC}}\right) \\
+\frac{3}{5} \mu v_{\mathrm{HC}}^{5 / 2}\left(2-3 v_{\mathrm{HC}}\right)\left(1-v_{\mathrm{HC}}\right)^{1 / 2}-\frac{3}{8} \mu^{2} v_{\mathrm{HC}}^{4}
\end{gathered}
$$

Conversely, when ohmic effects are large a closed-form solution has seen to be available for $v_{\mathrm{HC}}$ not exceeding $v_{\mathrm{HC} \text {,max }}$ given by Eq. (87), in which case

$$
\xi_{B}^{L}\left(v_{\mathrm{HC}}<v_{\mathrm{HC}, \max }\right)=\xi_{B, 1}^{L}=1-\frac{4 v_{\mathrm{HC}}^{1 / 4}}{\sqrt{\mu \epsilon}}
$$

For higher values of $v_{\mathrm{HC}}$ or for insulated tethers one has to make use of Eq. (17) where $\Omega$ is set to zero and the cathodic end potential is given by Eq. (94). A fourth-degree polynomial in $\xi_{B}$ is obtained:

$$
a \xi_{B}^{4}+b \xi_{B}^{3}+c \xi_{B}^{2}+d \xi_{B}+e=0
$$




$$
\begin{aligned}
& a=-\frac{1}{\hat{\xi}^{3}}+\frac{\mu \epsilon}{320} \cdot \frac{5 \hat{\xi}^{4}-60 \hat{\xi}^{3}-90 \hat{\xi}^{2}+20 \hat{\xi}-3}{\hat{\xi}^{4}}-\frac{\mu^{2} \epsilon^{2}}{40} \\
& b=\frac{1+3 \hat{\xi}}{\hat{\xi}^{3}}+\frac{\mu \epsilon}{40} \cdot \frac{5 \hat{\xi}^{3}+45 \hat{\xi}^{2}+15 \hat{\xi}-1}{\hat{\xi}^{3}}+\frac{\mu^{2} \epsilon^{2}}{10} \\
& c=-\frac{3+3 \hat{\xi}}{\hat{\xi}^{2}}-\frac{3 \mu \epsilon}{20} \cdot \frac{5 \hat{\xi}^{2}+10 \hat{\xi}+1}{\hat{\xi}^{2}}-\frac{3 \mu^{2} \epsilon^{2}}{20} \\
& d=\frac{3+\hat{\xi}}{\hat{\xi}}+\frac{\mu \epsilon}{5} \cdot \frac{5 \hat{\xi}+3}{\hat{\xi}}+\frac{\mu^{2} \epsilon^{2}}{10} \\
& e=-1+v_{\mathrm{HC}}-\frac{2 \mu \epsilon}{5}-\frac{\mu^{2} \epsilon^{2}}{40}
\end{aligned}
$$

Equation (100), to be solved numerically, provides the sought value $\xi_{B}^{L}\left(v_{\mathrm{HC}}>v_{\mathrm{HC}, \max }\right)=\xi_{B, 2}^{L}$.

After a simple numerical test one can see that the best estimate for $\xi_{B}$ across the whole $\left(v_{\mathrm{HC}}, \epsilon\right)$ parameter space is

$$
\begin{aligned}
& \xi_{B}\left(v_{\mathrm{HC}}, \Omega=0\right) \\
& = \begin{cases}\min \left\{\xi_{B}^{S}, \xi_{B, 2}^{L}\right\} & 0.05<v_{H C}<0.25 \\
\xi_{B}^{S} & v_{\mathrm{HC}}<0.05 \text { and } 0<\epsilon<\frac{8}{5 \xi_{B}^{3 / 2}} \\
\xi_{B, 1}^{L} & v_{\mathrm{HC}}<v_{\mathrm{HC}, \max } \text { and } \epsilon>\frac{8}{5 \xi_{B}^{3 / 2}} \\
\xi_{B, 2}^{L} & v_{\mathrm{HC}, \text { max }}<v_{\mathrm{HC}}<0.05 \text { and } \epsilon>\frac{8}{5 \xi_{B}^{3 / 2}}\end{cases}
\end{aligned}
$$

For $v_{\mathrm{HC}}<0.1$ the accuracy of the computed $\xi_{B}$ is high enough to allow a maximum error in the average current of less than about $7 \%$. For higher values of $v_{\mathrm{HC}}$ the error increases so that for $v_{\mathrm{HC}}>0.25$ the provided formula should not be used, with the exception of very low ohmic effects $(\epsilon \lesssim 1)$ in which sufficient accuracy is retained for practically any value of $v_{\mathrm{HC}}$.

The case of $v_{\mathrm{HC}}>0.1$ is of little practical interest as it corresponds to poorly designed EDTs in which the excessively high cathodic contactor impedance compromises the performance of the system. A comparison between the approximate analytical solution and the numerically-computed one is provided in a later section of the article.

\section{EDT Working Beyond the OML Regime}

When magnetic guiding effects are small, as it is the case in Earth orbit, OML current collection theory is valid for tethers whose equivalent radius $^{\dagger \dagger}$ does not exceed the local plasma Debye length $\lambda_{D}$ or a fraction of the latter depending on the local ion/electron plasma temperature ratio $[2,10]$. In Low Earth orbit the plasma Debye length reaches a minimum value of about three millimeters in daytime conditions which means that, for instance, tape tethers up to about $1 \mathrm{~cm}$ in width will collect OML current. Note, however, that if one neglects the small influence $[2,10]$ of the varying bias along the tether length the present analysis can be extended to the case in which the tether works beyond the OML regime. This is done by including a scaling factor, $G$, which is a function of the tether cross section, the local plasma Debye length and the local ion/electron temperature ratio and can be derived from the two references cited above. In this fashion, the modified characteristic current to take into account electrical effects reads

$$
\tilde{I}_{\mathrm{ch}} \sim G\left(\frac{R}{\lambda_{D}}, \frac{T_{i}}{T_{e}}\right) \cdot I_{\mathrm{ch}}
$$

where $G \leqslant 1$ is the current ratio $I / I_{\mathrm{OML}}$ given by Estes and Sanmartin $[10]$ as a function of the ratio $R / \lambda_{D}$ between the equivalent tether radius and the local plasma Debye length and the ratio $T_{i} / T_{e}$ between the local electron and ion temperatures.

\footnotetext{
${ }^{\dagger}$ For a thin tape the equivalent radius is equal to one-fourth of the tape width.
}

\section{Computing Lorentz Force/Torque and Generated Power}

Once an accurate solution has been found for the current and potential profile one can finally compute all the quantities relevant to tether operation. These are the average current, which provides the resulting Lorentz force, the first moment of the current, associated with the resulting Lorentz torque on the tether and the power generated at the load placed before the plasma contactor at the cathodic end.

\section{A. Average Current and Lorentz Force}

The average nondimensional current along the tether line is

$$
i_{\mathrm{av}}=\int_{0}^{\xi_{B}} i_{a} \mathrm{~d} \xi+\int_{\xi_{B}}^{1} i_{c} \mathrm{~d} \xi
$$

which for the case of small ohmic effects [Eqs. (55) and (56)] yields

$$
i_{\mathrm{av}}^{S}=i_{\mathrm{av} 0}+\epsilon i_{\mathrm{av} 1}+\epsilon^{2} \dot{l}_{\mathrm{av} 2}
$$

where

$$
\begin{gathered}
i_{\mathrm{av} 0}=\frac{\xi_{B}^{3 / 2}\left(5-2 \xi_{B}\right)-2 \mu\left(1-\xi_{B}\right)^{5 / 2}}{5} \\
i_{\mathrm{av} 1}=\frac{1}{40}\left[7 \xi_{B}^{4}-16 \xi_{B}^{3}+8 \mu \xi_{B}^{3 / 2}\left(1-\xi_{B}\right)^{5 / 2}-\mu^{2}\left(1-\xi_{B}\right)^{4}\right] \\
i_{\mathrm{av} 2}=\frac{1}{4400}\left[\xi_{B}^{9 / 2}\left(319-130 \xi_{B}\right)-132 \mu \xi_{B}^{3}\left(1-\xi_{B}\right)^{5 / 2}\right. \\
\left.+2 \mu^{3}\left(1-\xi_{B}\right)^{11 / 2}\right]
\end{gathered}
$$

For the case of large ohmic effect we integrate Eqs. (75), (77), (82), (83), (88), and (95) to obtain

$$
\begin{aligned}
& i_{\mathrm{av}}^{L}\left(\xi_{B}<\hat{\xi}\right)=i_{\mathrm{av}, 1}^{L} \\
& =\frac{\xi_{B}^{2}\left(\xi_{B}^{2}-4 \xi_{B} \hat{\xi}+6 \hat{\xi}^{2}\right)+4\left(1-\xi_{B}\right)\left(\xi_{B}^{3}-3 \xi_{B}^{2} \hat{\xi}+3 \xi_{B} \hat{\xi}^{2}\right)}{4 \epsilon \hat{\xi}^{3}} \\
& -\frac{2}{5} \mu \frac{\left(\hat{\xi}-\xi_{B}\right)^{3 / 2}\left(1-\xi_{B}\right)^{5 / 2}}{\hat{\xi}^{3 / 2}}-\frac{1}{40} \mu^{2} \epsilon\left(1-\xi_{B}\right)^{4} \\
& i_{\mathrm{av}}^{L}\left(\xi_{B} \geqslant \hat{\xi}\right)=i_{\mathrm{av}, 2}^{L}=\frac{4-\hat{\xi}}{4 \epsilon}-\frac{\mu^{2} \epsilon}{64}\left(1-\xi_{B}\right)^{4}
\end{aligned}
$$

The average current can then be written in the following compact form:

$$
i_{\mathrm{av}}=\frac{\xi_{B}^{3 / 2}\left(5-2 \xi_{B}\right)-2 \mu\left(1-\xi_{B}\right)^{5 / 2}}{5} \eta_{\mathrm{th}}
$$

where we have introduced the thrust ohmic efficiency:

$$
\eta_{\mathrm{th}}=\frac{i_{\mathrm{av}}}{i_{\mathrm{av}}(\epsilon=0)}= \begin{cases}\frac{i_{\mathrm{av} 0}+\epsilon i_{\mathrm{av} 1}+\epsilon^{2} i_{\mathrm{av} 2}}{i_{\mathrm{av} 0}} & 0<\epsilon<\frac{8}{5 \xi_{B}^{3 / 2}} \\ \frac{i_{\mathrm{av}, 1}^{L}}{i_{\mathrm{av} 0}} & \frac{8}{5 \xi_{B}^{3 / 2}}<\epsilon<\frac{4}{\xi_{B}^{3 / 2}} \\ \frac{i_{\mathrm{av}, 2}^{L}}{i_{\mathrm{av} 0}} & \epsilon>\frac{4}{\xi_{B}^{3 / 2}}\end{cases}
$$

The above formulas simplify considerably for the particular case of zero load and negligible potential drop at the cathodic contactor $\left(\Omega=v_{\mathrm{HC}}=0\right)$. In such case $\xi_{B}=1$, the average current is maximum and yields 


$$
i_{\mathrm{av}}\left(\xi_{B}=1\right)=\frac{3}{5} \eta_{\mathrm{th}}
$$

with

$$
\eta_{\mathrm{th}}\left(\xi_{B}=1\right)= \begin{cases}1-\frac{3}{8} \epsilon+\frac{63}{880} \epsilon^{2} & 0<\epsilon<1.6 \\ \frac{5}{3 \epsilon}\left(1-\frac{1}{(2 \epsilon)^{2 / 3}}\right) & \epsilon>1.6\end{cases}
$$

Once the nondimensional average current is known the Lorentz force can be readily computed as

$$
\mathbf{F}=i_{\mathrm{av}} I_{\mathrm{ch}} B L\left(\mathbf{u}_{t} \wedge \mathbf{u}_{B}\right)
$$

where $B$ is the magnetic field intensity while $\mathbf{u}_{\mathbf{t}}$ and $\mathbf{u}_{B}$ are, respectively, the tether line unit vector directed along the current flow and the magnetic field unit vector. It is important to stress that if the EDT has only one cathodic plasma contactor placed at one tether end a nonnegligible current will flow only if the projection of the local electric field along the tether is oriented towards the same cathodic plasma contactor.

A final design parameter of interest for EDT missions is the specific thrust. The latter is defined as the ratio between the magnitude of the Lorentz force and the tether mass, and can be written taking into account Eqs. (14) and (110):

$$
\frac{F}{m}=\frac{i_{\mathrm{av}} I_{\mathrm{ch}} B L\left|\mathbf{u}_{t} \wedge \mathbf{u}_{B}\right|}{\rho A L} \propto \epsilon i_{\mathrm{av}}
$$

The latter quantity is plotted in Fig. 5, for the case of zero load and zero potential drop at the cathode, utilizing Eq. (109). The plots confirms a key aspect, already known in the literature ([3]), regarding bare EDTs: best use of tether mass is made by pushing the design towards the dominant ohmic effect regime, i.e., employing longer tethers with smaller thickness (for tape tethers) or radius (for cylindric tethers).

\section{B. Lorentz Torque}

The (nondimensional) distance of the point of application of the force from the anodic end of the tether is

$$
\xi_{d}=\frac{1}{i_{\mathrm{av}}}\left(\int_{0}^{\xi_{B}} \xi i_{A} \mathrm{~d} \xi+\int_{\xi_{B}}^{1} \xi i_{C} \mathrm{~d} \xi\right)
$$

which for the case of small ohmic effects results in

$$
\xi_{d}^{S}=\xi_{d 0}^{S}+\epsilon \xi_{d 1}^{S}+\epsilon^{2} \xi_{d 2}^{S}+\mathcal{O}\left(\epsilon^{2}\right)
$$

where

$$
\xi_{d 0}^{S}=\frac{1}{14} \frac{8 \xi_{B}^{7 / 2}-35 \xi_{B}^{3 / 2}+4 \mu\left(1-\xi_{B}\right)^{5 / 2}\left(5+2 \xi_{B}\right)}{2 \xi_{B}^{5 / 2}-5 \xi_{B}^{3 / 2}+2 \mu\left(1-\xi_{B}\right)^{5 / 2}}
$$

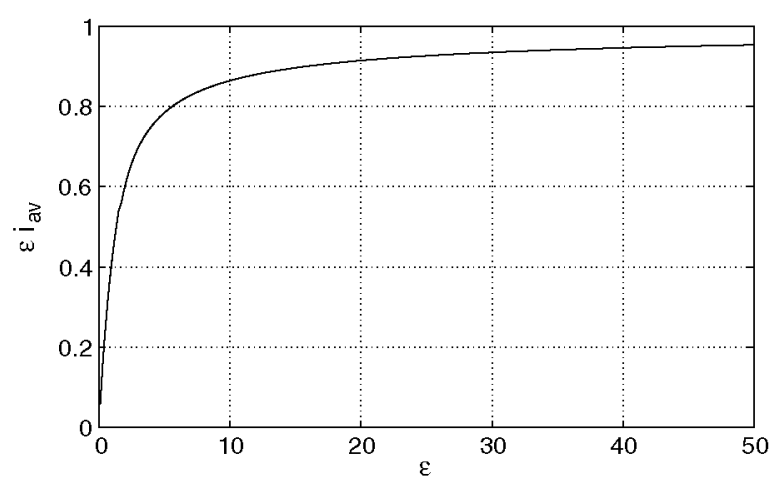

Fig. 5 Variation of the quantity $\epsilon i_{\mathrm{av}}$, which is proportional to the EDT specific thrust, as a function of the parameter $\epsilon$. No load is inserted at the cathodic end of the tether and the cathode potential drop is neglected.

$$
\begin{aligned}
\xi_{d 1}^{S} & =-\frac{3}{560} \frac{\xi_{B}^{5 / 2}\left(4 \xi_{B}^{2}-30 \xi_{B}+35\right)}{\left(2 \xi_{B}-5\right)^{2}} \\
& +\frac{3 \mu}{140} \frac{\left(1-\xi_{B}\right)^{5 / 2}\left(2 \xi_{B}^{3}-15 \xi_{B}^{2}+30 \xi_{B}-50\right)}{\left(2 \xi_{B}-5\right)^{3}}+\mathcal{O}\left(\mu^{2}\right) \\
\xi_{d 2}^{S} & =-\frac{3 \xi_{B}^{4}}{640640} \frac{3748 \xi_{B}^{3}-36238 \xi_{B}^{2}+95003 \xi_{B}-72800}{\left(2 \xi_{B}-5\right)^{3}}+\mathcal{O}(\mu)
\end{aligned}
$$

Conversely, in the case of large ohmic effects we have

$$
\xi_{d}^{L}\left(\xi_{B} \geqslant \hat{\xi}\right)=\frac{1}{5} \frac{16\left(\hat{\xi}^{2}-10\right)+\mu^{2} \epsilon^{2}\left(4+\xi_{B}\right)\left(1-\xi_{B}\right)^{4}}{16(\hat{\xi}-4)+\mu^{2} \epsilon^{2}\left(1-\xi_{B}\right)^{4}}
$$

and

$$
\xi_{d}^{L}\left(\xi_{B}<\hat{\xi}\right)=\xi_{d 0}^{L}+\mu \xi_{d 1}^{L}+\mathcal{O}(\mu)
$$

where

$$
\xi_{d 0}^{L}=\frac{1}{5} \frac{10\left(1-\xi_{B}^{2}\right)\left(\xi_{B}^{3}-3 \xi_{B}^{2} \hat{\xi}+3 \xi_{B} \hat{\xi}^{2}\right)+\xi_{B}^{3}\left(20 \hat{\xi}^{2}-15 \xi_{B} \hat{\xi}+4 \xi_{B}^{2}\right)}{4\left(1-\xi_{B}\right)\left(\xi_{B}^{3}-3 \xi_{B}^{2} \hat{\xi}+3 \xi_{B} \hat{\xi}^{2}\right)+\xi_{B}^{2}\left(6 \hat{\xi}^{2}-4 \xi_{B} \hat{\xi}+\xi_{B}^{2}\right)}
$$

$$
\begin{aligned}
\xi_{d 1}^{L} & =\frac{8 \epsilon^{2}}{175} \frac{\left(1-\xi_{B}\right)^{5 / 2}\left(\hat{\xi}-\xi_{B}\right)^{3 / 2} \hat{\xi}^{3}\left(3 \xi_{B}^{3}-8 \xi_{B}^{2} \hat{\xi}+6 \xi_{B} \hat{\xi}^{2}-4 \xi_{B}^{2}+12 \xi_{B} \hat{\xi}-12 \hat{\xi}^{2}\right)}{} \\
& \times\left[30 \xi_{B}^{2}-90 \xi_{B} \hat{\xi}+90 \hat{\xi}^{2}+12 \xi_{B}^{4}-25 \xi_{B}^{3} \hat{\xi}+10 \xi_{B}^{2} \hat{\xi}^{2}\right. \\
& \left.-35 \xi_{B}^{3}+80 \xi_{B}^{2} \hat{\xi}-30 \xi_{B} \xi^{2}\right] .
\end{aligned}
$$

Once $\xi_{d}$ is known the torque about the tether center of mass $G$ can be computed as

$$
\tau=I_{\mathrm{ch}} i_{\mathrm{av}} B L^{2}\left(\xi_{d}-\xi_{G}\right)\left[\mathbf{u}_{t} \wedge\left(\mathbf{u}_{t} \wedge \mathbf{u}_{B}\right)\right]
$$

where $\xi_{G}$ is the dimensionless abscissa of the center of mass.

\section{Generated Power}

The power generated at the load in nondimensional form can be computed as

$$
\dot{w}=-i_{c}(1) \varphi_{c}(1)
$$

Using Eqs. (42) and (46) we obtain the power generated for the small-ohmic-effect case:

$$
\dot{w}^{S}=\dot{w}_{0}+\epsilon \dot{w}_{1}+\epsilon^{2} \dot{w}_{2}+\epsilon^{3} \dot{w}_{3}+\epsilon^{4} \dot{w}_{4}+\epsilon^{5} \dot{w}_{5}
$$

with

$$
\begin{gathered}
\dot{w}_{0}=\xi_{B}^{3 / 2}\left(1-\xi_{B}\right)-\mu\left(1-\xi_{B}\right)^{5 / 2} \\
\dot{w}_{1}=-\frac{7}{5} \xi_{B}^{3}\left(1-\xi_{B}\right)+\frac{19}{10} \mu \xi_{B}^{3 / 2}\left(1-\xi_{B}\right)^{5 / 2}-\frac{\mu^{2}}{2}\left(1-\xi_{B}\right)^{4} \\
\dot{w}_{2}=\frac{349}{400} \xi_{B}^{9 / 2}\left(1-\xi_{B}\right)-\frac{267}{200} \mu \xi_{B}^{3}\left(1-\xi_{B}\right)^{5 / 2} \\
+\frac{21}{40} \mu^{2} \xi_{B}^{3 / 2}\left(1-\xi_{B}\right)^{4} \\
\dot{w}_{3}=-\frac{61}{200} \xi_{B}^{6}\left(1-\xi_{B}\right)+\frac{973}{2000} \mu \xi_{B}^{9 / 2}\left(1-\xi_{B}\right)^{5 / 2} \\
-\frac{21}{100} \mu^{2} \xi_{B}^{3}\left(1-\xi_{B}\right)^{4}
\end{gathered}
$$




$$
\begin{aligned}
\dot{w}_{4} & =\frac{29}{500} \xi_{B}^{15 / 2}\left(1-\xi_{B}\right)+\frac{371}{4000} \mu \xi_{B}^{6}\left(1-\xi_{B}\right)^{5 / 2} \\
& +\frac{5}{128} \mu^{2} \xi_{B}^{9 / 2}\left(1-\xi_{B}\right)^{4} \\
\dot{w}_{5} & =-\frac{841}{160000} \xi_{B}^{9}\left(1-\xi_{B}\right)+\frac{609}{80000} \mu \xi_{B}^{15 / 2}\left(1-\xi_{B}\right)^{5 / 2} \\
& -\frac{9}{4000} \mu^{2} \xi_{B}^{6}\left(1-\xi_{B}\right)^{4}
\end{aligned}
$$

where we have neglected terms of order $\mu^{2}$ and higher.

For the case of large ohmic effects [Eqs. (94) and (95)] we obtain

$$
\begin{gathered}
\dot{w}^{L}\left(\xi_{B}<\hat{\xi}\right)=\dot{w}_{1}^{L}=\frac{\left(1-\xi_{B}\right)\left(\hat{\xi}-\xi_{B}\right)^{3}\left(\xi_{B}^{3}-3 \xi_{B}^{2} \hat{\xi}+3 \xi_{B} \hat{\xi}^{2}\right)}{\epsilon \hat{\xi}^{6}} \\
-\frac{\mu}{5} \frac{\left(\hat{\xi}-\xi_{B}\right)^{3 / 2}\left(5 \hat{\xi}^{3}-21 \hat{\xi}^{2} \xi_{B}+21 \hat{\xi} \xi_{B}^{2}-7 \xi_{B}^{3}\right)\left(1-\xi_{B}\right)^{5 / 2}}{\hat{\xi}^{9 / 2}} \\
-\frac{\mu^{2} \epsilon}{40} \frac{\left(20 \hat{\xi}^{3}-63 \hat{\xi}^{2} \xi_{B}+63 \hat{\xi} \xi_{B}^{2}-21 \xi_{B}^{3}\right)\left(1-\xi_{B}\right)^{4}}{\hat{\xi}^{3}}
\end{gathered}
$$

and

$$
\dot{w}^{L}\left(\xi_{B} \geqslant \hat{\xi}\right)=\dot{w}_{2}^{L}=\frac{\mu^{2} \epsilon}{64}\left(1-\xi_{B}\right)^{4}-\frac{\mu^{4} \epsilon^{3}}{1024}\left(1-\xi_{B}\right)^{7}
$$

The generated power can then be written in the following compact form:

$$
\dot{w}=\left[\xi_{B}^{3 / 2}\left(1-\xi_{B}\right)-\mu\left(1-\xi_{B}\right)^{5 / 2}\right] \cdot \eta_{w}
$$

where we have introduced the power ohmic efficiency:

$$
\eta_{w}=\frac{\dot{w}}{\dot{w}(\epsilon=0)}= \begin{cases}\frac{\dot{w}_{0}+\epsilon \dot{w}_{0}+. .+\epsilon^{5} \dot{w}_{5}}{\dot{w}_{0}} & 0<\epsilon<\frac{8}{5 \xi_{B}^{3 / 2}} \\ \frac{\dot{w}_{1}^{L}}{\dot{w}_{0}} & \frac{8}{5 \xi_{B}^{3 / 2}}<\epsilon<\frac{4}{\xi_{B}^{3 / 2}} \\ \frac{\dot{w}_{2}^{L}}{\dot{w}_{0}} & \epsilon>\frac{4}{\xi_{B}^{3 / 2}}\end{cases}
$$

Let us now search for the a possible value of the control parameter $\xi_{B}$ providing maximum power, hence obeying

$$
\frac{\mathrm{d} \dot{w}}{\mathrm{~d} \xi_{B}}\left(\xi_{B}^{\mathrm{opt}}\right)=0
$$

For small ohmic effects Eq. (134) yields a nonlinear equation not solvable analytically. In the range $0<\epsilon<1.5$ an accurate solution (within $2 \%$ of relative error) of Eq. (134) can be again obtained with a perturbation method:

$$
\begin{aligned}
& \left(\xi_{B}^{\text {opt }}\right)^{S} \approx \frac{3}{5}+\frac{2 \sqrt{6}}{15} \mu-\frac{22}{45} \mu^{2}+\epsilon\left(-\frac{126 \sqrt{15}}{3125}+\frac{54 \sqrt{10}}{625} \mu\right) \\
& +\epsilon^{2} \frac{3483}{312500}
\end{aligned}
$$

For higher values of $\epsilon$ Eq. (134) must be handled numerically. The corresponding curve is plotted in Fig. 4 (dashed line). As $\epsilon$ increases the corresponding $\xi_{B}^{\text {opt }}$ decreases in such a way that the current profile always remains in the low ohmic effect region. An inspection of the exact numerical solution of the current and potential profile confirms that the power profile as a function of $\xi_{B}$ has only one maximum, which corresponds to the aforementioned curve. All in all we can conclude that optimum power generation always occurs in the small ohmic effects regime.
The corresponding dimensional power generated at the load can be finally computed as

$$
\dot{W}_{\ell}=\dot{w} I_{\mathrm{ch}} E_{t} L
$$

\section{Traditional Formulation}

The formulation of the bias and current profile commonly employed in the literature (see for instance [3]) refers to different dimensionless quantities, which are here denoted with a tilde superscript. Next we will provide conversion formulas between the two formulations. In the traditional formulation the nondimensionalization of the current is done with respect to the short-circuit value so that

$$
\tilde{i}=\frac{I}{\sigma A E_{t}}=\epsilon i
$$

As far as the length a characteristic reference value $L^{*}$ is used in the literature:

$$
L^{*}=\left(\frac{9 \pi^{2} m_{e} \sigma^{2} E_{t} A^{2}}{32 q_{e} N_{e}^{2} p^{2}}\right)^{1 / 3}
$$
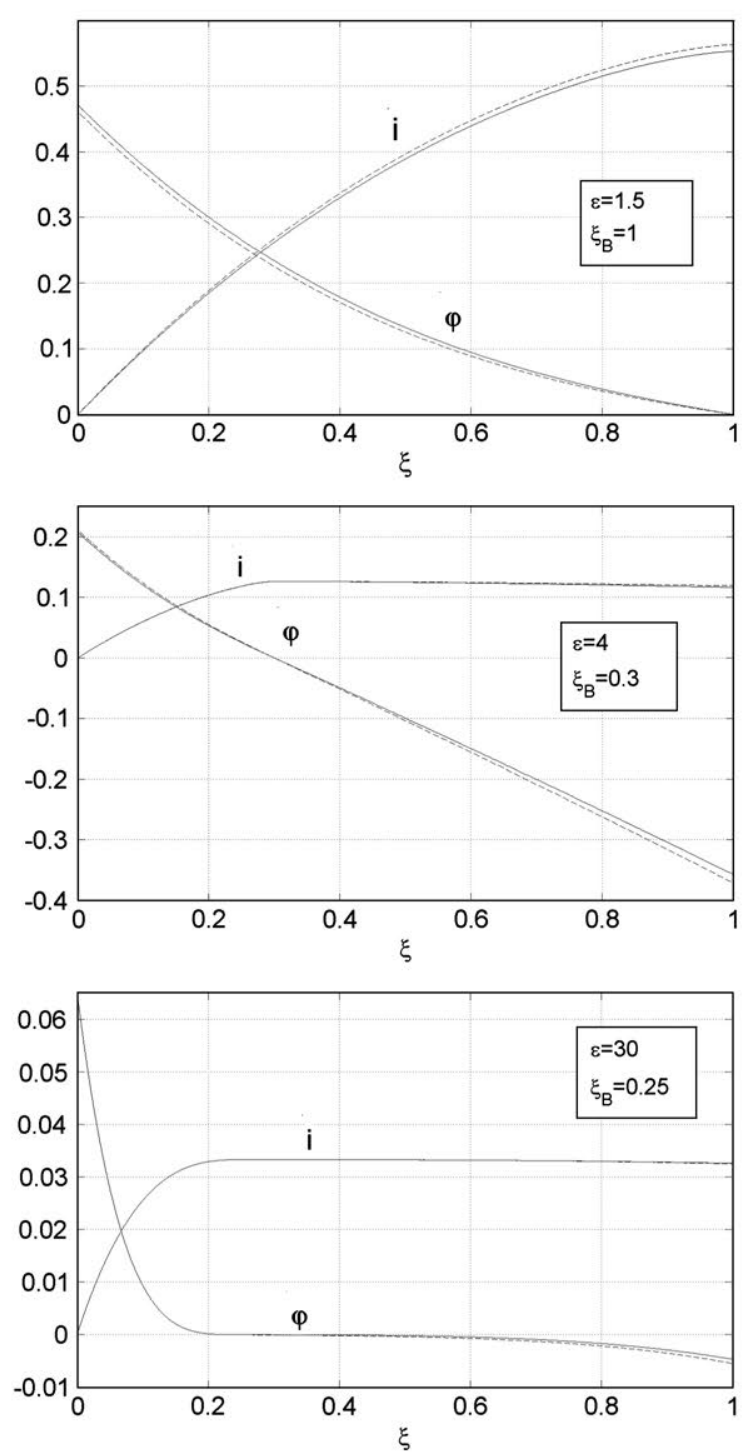

Fig. 6 Comparison between dimensionless current and local bias profile obtained numerically (solid line) and with the proposed analytical method (dash-line) for different values of $\epsilon$ and $\xi_{B}$. The coefficient $\mu$ has been set to $1 / 43$. 


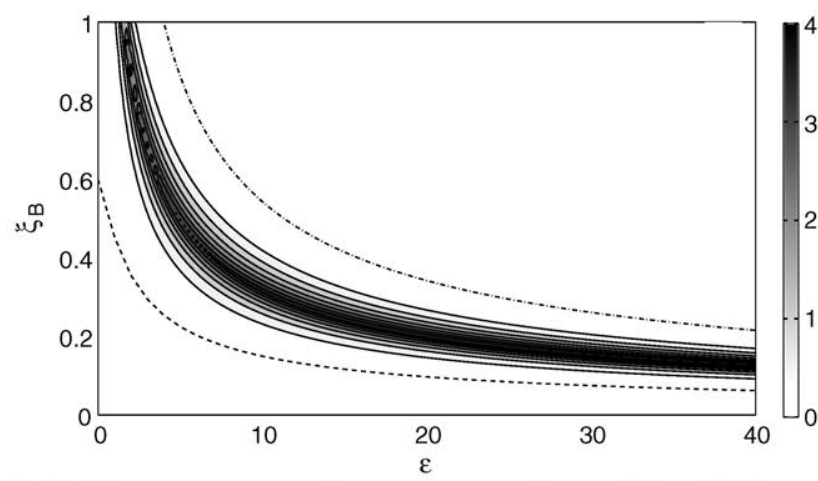

Fig. 7 Percentage error between approximate [Eq. (132)] and numerical solution for the dimensionless average current. The parameter $\mu$ is here set to zero (insulated cathodic segment). The lower dash-line corresponds to optimal power generation. The upper dash-dot line represents the condition $\xi_{B}=\hat{\xi}$. Note that in this case $(\mu=0)$ the condition $\xi_{B}>\hat{\xi}$ cannot be reached. The case of $\mu \neq 0$ produces a very similar plot.

Our parameter $\epsilon$ can be directly related to the ratio $L / L^{*}$ using Eqs. (27) and (138):

$$
\epsilon=\frac{1}{2}\left(\frac{L}{L^{*}}\right)^{3 / 2}
$$

The conversion of the nondimensional abscissa with our new formulation yields

$$
\tilde{\xi}=\frac{x}{L^{*}}=\frac{L}{L^{*}} \xi=(2 \epsilon)^{2 / 3} \xi
$$

and as a particular case our parameter $\xi_{B}$ can be written as

$$
\xi_{B}=\frac{\tilde{\xi}_{B} L^{*}}{L}=\frac{\tilde{\xi}_{B}}{(2 \epsilon)^{2 / 3}}
$$

Similarly for the potential we have

$$
\tilde{\varphi}=\frac{\Delta V}{E_{t} L^{*}}=\frac{L}{L^{*}} \varphi=(2 \epsilon)^{2 / 3} \varphi
$$

\section{Global Solution and Accuracy}

To test the accuracy of the proposed method an extensive test campaign has been conducted in which our analytical solution is compared with the numerical solution of the current and local bias equations employing a shooting method. Figure 6 compares current and potential profile curves for different $\left(\xi_{B}, \epsilon\right)$ configurations corresponding to low and high ohmic effects. The error remains very small across the whole profile.

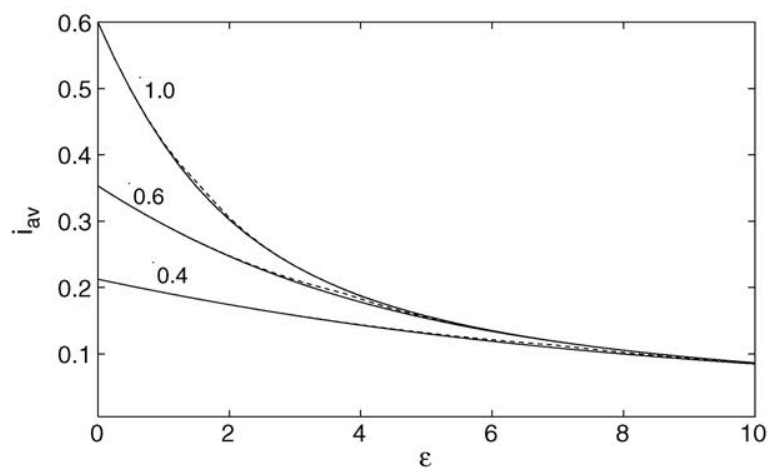

Fig. 8 Comparison between analytically- (dash-line) and numericallycomputed (solid line) dimensionless average current as a function of the parameter $\epsilon$ and for three different values of the coefficient $\xi_{B}$. The coefficient $\mu$ is here set to $1 / 43$.

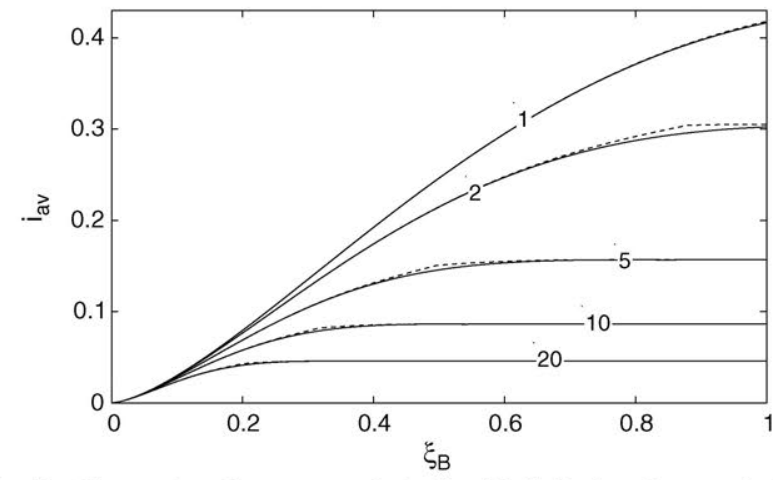

Fig. 9 Comparison between analytically- (dash-line) and numericallycomputed (solid line) dimensionless average current as a function of the parameter $\xi_{B}$ and for five different values of the coefficient $\epsilon$. The coefficient $\mu$ is here set to $1 / 43$.

Figure 7 plots the error between the analytically and the numerically-computed average current across a relatively large span of the $\left(\epsilon, \xi_{B}\right)$ parameter space. The maximum error does not exceed $5 \%$ demonstrating that the present method is particularly effective for the computation of the average current. This can also be seen in Figs. 8 and 9 where we compare the average current computed with the two methods for varying $\epsilon$ and $\xi_{B}$.

Figure 10 plots the error between the analytically and the numerically-computed generated power across a relatively large span of the $\left(\epsilon, \xi_{B}\right)$ parameter space. In this case the maximum error can reach up to $35 \%$. However, the poor-accuracy region is of little practical interest as it corresponds to values of the parameters $\xi_{B}$ and $\epsilon$

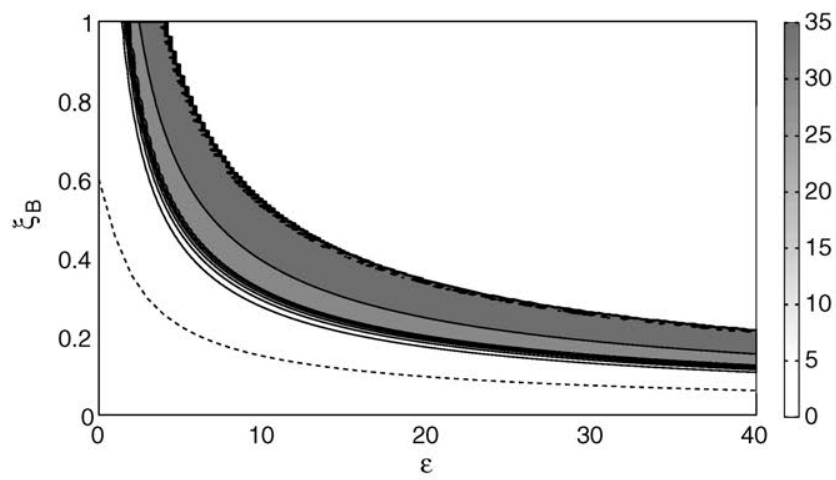

Fig. 10 Percentage error between approximate [Eq. (132)] and numerical solution for the generated power. The parameter $\mu$ is here set to zero (insulated cathodic segment). The lower dash-line corresponds to optimal power generation. The case of $\mu \neq 0$ produces a very similar plot.

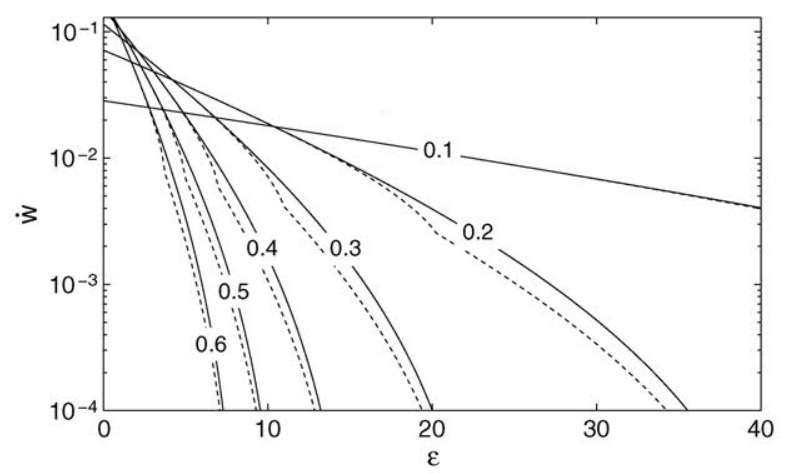

Fig. 11 Comparison between analytically- (dash-line) and numerically-computed (solid line) dimensionless generated power as a function of the parameter $\epsilon$ and for six different values of the coefficient $\xi_{B}$. The coefficient $\mu$ is here set to zero. 


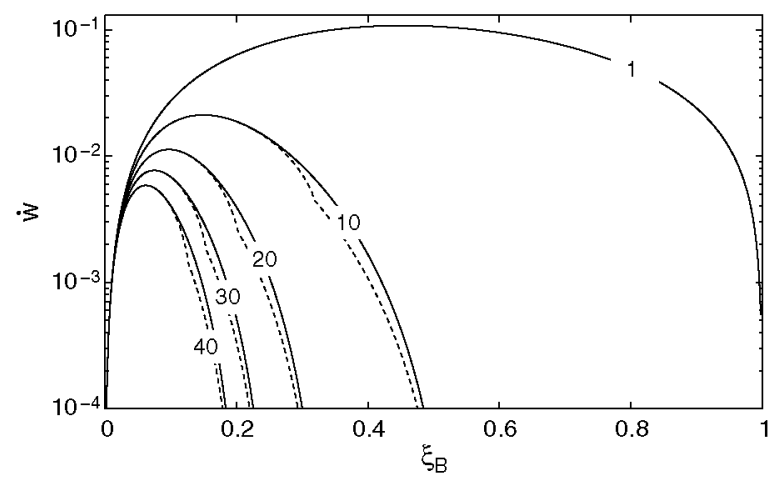

Fig. 12 Comparison between analytically- (dash-line) and numerically-computed (solid line) dimensionless generated power as a function of the parameter $\xi_{B}$ and for five different values of the coefficient $\epsilon$. The coefficient $\mu$ is here set to zero. Note the existence of an optimum value of $\xi_{B}$ for maximum power and the negligibly small error obtained around optimal conditions.

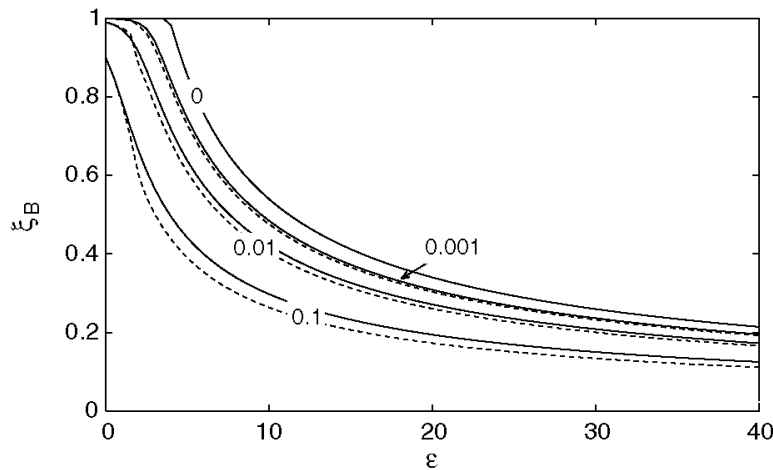

Fig. 13 Comparison between dimensionless anodic segment length computed analytically through Eq. (101) (dash-line) and numerically for varying $\epsilon$, zero load $(\Omega=0)$ and four different values of the dimensionless potential drop at the cathode $\left(v_{\mathrm{HC}}\right)$. In this example the cathodic segment is insulated $(\mu=0)$. Note that for $v_{\mathrm{HC}}=0$ we have $\xi_{B} \equiv \hat{\xi}$.

which are far from the optimum power generation condition given by Eq. (134). Conversely, the error obtained under maximum power generation condition is negligible. This can also be seen in Figs. 11 and 12 where we compare the generated power computed with the two methods for varying $\epsilon$ and $\xi_{B}$.

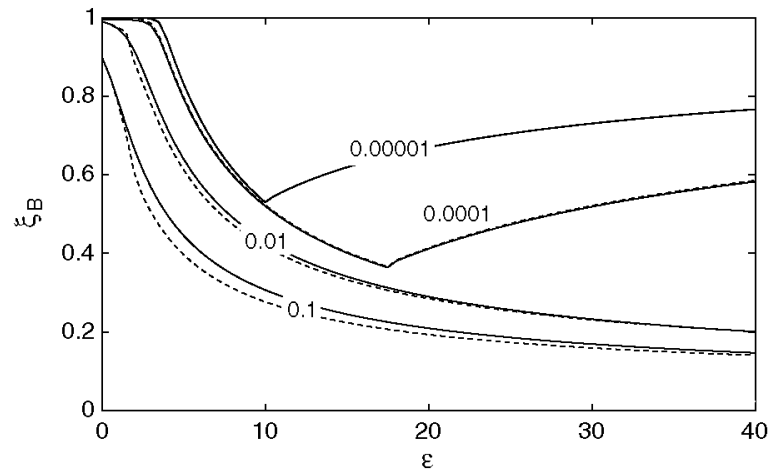

Fig. 14 Comparison between dimensionless anodic segment length computed analytically through Eq. (101) (dash-line) and numerically for varying $\epsilon$, zero load $(\Omega=0)$ and four different values of the dimensionless potential drop at the cathode $\left(v_{\mathrm{HC}}\right)$. In this example the uninsulated cathodic segment $(\mu=1 / 43)$ makes it possible to have $\xi_{B}>\hat{\xi}$ for sufficiently small $v_{\mathrm{HC}}$ and high $\epsilon$, in which case $\xi_{B}$ reaches a minimum to then grow beyond $\hat{\xi}$.

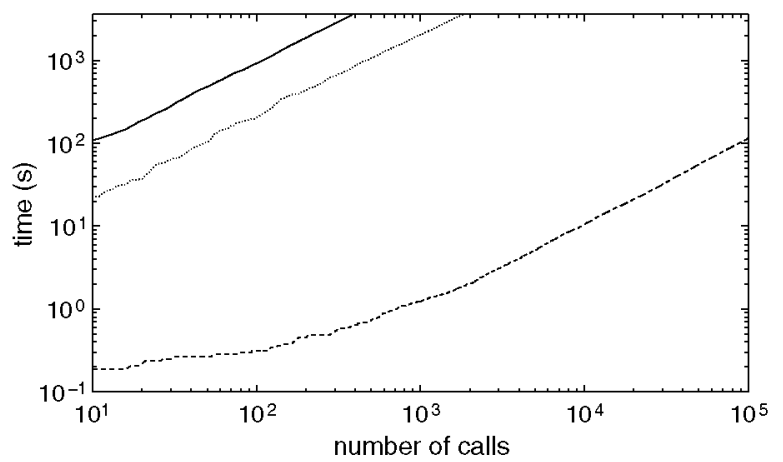

Fig. 15 Average current computation time for the three different methods: analytical (dash), semi-analytical (dot) and numerical (solid).

Figures 13 and 14 compare the numerically and analytically computed dimensionless anodic segment length $\xi_{B}$ as a function of the parameter $\epsilon$ and for different values of the plasma contactor potential drop $v_{\mathrm{HC}}$. The error remain relatively small as long as $v_{\mathrm{HC}}$ is not too high, which is reasonable for practical EDT applications.

As far as the Lorentz torque is concerned, the relative error, not reported here, between the exact and approximate solution does not exceed $1 \%$ across the whole parameter space.

Finally, the performance of the provided solution in terms of computation speed has been compared with the one computed numerically with a standard shooting method and with the method provided by [5]. As can be seen in Fig. 15 the analytical solution provides more than 3 orders of magnitude improvement in computation time.

\section{Conclusions}

This paper provided an accurate analytical formulation describing the current and potential profile for passive bare EDTs in the OML regime. The compact analytical formulas employ two nondimensional parameters $\epsilon$ and $\xi_{B}$. The first represents the ratio between tether contact and ohmic impedance, the second is the nondimensional voltage drop at the power generating load gauged by the electric field potential difference across the tether length. All quantities relevant to EDT mission design and operation can be computed directly with the provided formulas. The average current along the tether, and hence the resulting Lorentz force, can be estimated with accuracy of better than $5 \%$ across the whole parameter space. While the error on the evaluation of the generated power can be higher the low-accuracy region (going from a 5 to $30 \%$ maximum error) is of little interest for realEDT applications as it corresponds to a badly chosen value of the load impedance, providing low specific power and poor efficiency.

A comparison with existing numerical methods shows an improvement of more than three orders of magnitude in terms of computational speed showing that the proposed analytical solution can be advantageously employed for the numerical analysis of orbital and attitude control and dynamics involving bare EDTs.

\section{Acknowledgment}

The work was conducted in the framework of the research project "Propagation of Orbits, Advanced Orbital Dynamics and Use of Space Tethers," supported by the Dirección General de Investigación of the Spanish Ministry of Education and Science through the contract ESP2007-64068.

\section{References}

[1] Sanmartin, J. R., Ahedo, E., and Martinez-Sanchez, M., "An Anodeless Tether Generator," Proceedings of Physics of Charged Bodies in Space Plasmas, edited by M. Dobrowolny and E. Sindoni, 1991.

[2] Sanmartin, J. R., Lorenzini, E. C., and Martinez-Sanchez, M. "Electrodynamic Tether Applications and Constraints," Journal of Spacecraft and Rockets, Vol. 47, No. 3, 2010, pp. 442-456. 
[3] Sanmartin, J. R., Martinez-Sanchez, M., and Ahedo, E., "Bare Wire Anodes for Electrodynamic Tethers," Joumal of Propulsion and Power, Vol. 9, No. 3, 1993, pp. 353-360. doi: $10.2514 / 3.23629$

[4] Estes, R. D., Lorenzini, E. C., Sanmartin, J. R., Pelaez, J., MartinezSanchez, M., Johnson, C. L., and Vas, I. E., "Bare Tethers for Electrodynamic Spacecraft Propulsion," Joumal of Spacecraft and Rockets, Vol. 37, No. 2, 2000, pp. 205-211. doi: $10.2514 / 2.3567$

[5] Leamy, M. J., Noor, A. K., and Wasfy, T. M., "Sensitivity Analysis of Bare-Wire Space Tether Systems," Computer Methods in Applied Mechanics and Engineering, Vol. 190, No. 42, 2001, pp. 5495-5503. doi: $10.1016 / \mathrm{SO} 045-7825(01) 00163-3$

[6] Nayfeh, A., Introduction to Perturbation Techniques, WileyInterscience, New York, 1981.

[7] Sanmartin, J. R., Charro, M., Pelaez, J., Tinao, I., Elaskar, S., Hilgers, A., and Martinez-Sanchez, M., "Floating Bare Tether as Upper Atmosphere Probe,' Journal of Geophysical Research (Space Physics),
Vol. 111, No. A11, 2006 , p. A11310.

doi: $10.1029 / 2006$ JA011624

[8] Divine, N., and Garrett, H. B., "Charged Particle Distributions in Jupiter's Magnetosphere," Joumal of Geophysical Research, Vol. 88, No. A9, 1983, pp. 6889-6903. doi:10.1029/JA088iA09p06889

[9] Ahedo, E., and Sanmartin, J. R., "Analysis of Bare-Tether Systems for Deorbiting Low-Earth-Orbit Satellites," Joumal of Spacecraft and Rockets, Vol. 39, No. 2, 2002, pp. 198-205. doi: $10.2514 / 2.3820$

[10] Estes, R. D., and Sanmartin, J. R., "Cylindrical Langmuir Probes Beyond the Orbital-Motion-Limited Regime," Physics of Plasmas, Vol. 7, No. 10, 2000 , pp. 4320

doi: $10.1063 / 1.1288400$ 